\title{
«Femmine da conio». Una metafora monetaria
}

"Femmine da conio ». Une métaphore monétaire

"Femmine da conio". A Monetary Metaphor

\section{Sebastiana Nobili e Lucia Travaini}

\section{OpenEdition}

\section{Journals}

Edizione digitale

URL: https://journals.openedition.org/cei/9685

DOI: 10.4000/cei.9685

ISSN: 2260-779X

\section{Editore}

UGA Éditions/Université Grenoble Alpes

\section{Edizione cartacea}

ISBN: 978-2-37747-304-5

ISSN: $1770-9571$

\section{Notizia bibliografica digitale}

Sebastiana Nobili e Lucia Travaini, ««Femmine da conio». Una metafora monetaria», Cahiers d'études italiennes [Online], 33 | 2021, online dal 01 octobre 2021, consultato il 13 octobre 2021. URL: http:// journals.openedition.org/cei/9685; DOI: https://doi.org/10.4000/cei.9685

Questo documento è stato generato automaticamente il 13 octobre 2021

(C) ELLUG 


\title{
«Femmine da conio». Una metafora monetaria
}

\author{
"Femmine da conio ». Une métaphore monétaire \\ “Femmine da conio". A Monetary Metaphor
}

Sebastiana Nobili e Lucia Travaini

\section{NOTE DELL'AUTORE}

Nel preparare una Conversazione dantesca con Luigi Canetti a Ravenna nell'ottobre del 2018 Lucia Travaini esaminò gli scritti di Dante con riferimenti alle monete: sono molti e vanno dagli aspetti più negativi a quelli più positivi, arrivando inaspettatamente perfino alla metafora della Fede come buona moneta (Par. XXIV, 87). Tralasciò però di trattare pubblicamente in quella sede il riferimento a «femmine da conio» (Inf. XVIII, 66) perché se l'interpretazione era per lei - esperta di monete e zecche medievali - molto chiara, essa non lo era affatto per i dantisti le cui ipotesi aveva letto nella sintesi della voce 'conio' di Eugenio Ragni nell'Enciclopedia Dantesca del 1970. A Ravenna sperava quindi di incontrare esperti dantisti con cui analizzare insieme le «femmine da conio» di Dante, e con grande piacere incontrò Sebastiana Nobili che era presente e curiosa di vedere una nuova impostazione del tema. Da quel momento il quesito di Lucia Travaini è diventato lo studio congiunto che viene presentato nel contributo in questo volume. Per quanto riguarda più specificamente le due parti di cui si compone questo saggio, Sebastiana Nobili ha curato la parte 1, Lucia Travaini la parte 2: il lavoro è stato tuttavia progettato e discusso nella sua interezza dalle due autrici, in unità di metodo e di intenti.

\section{Ruffiani e sedotte}

1 Il XVIII dell'Inferno è un canto composito, in cui si parla delle donne ma dove le donne appaiono soprattutto nel ruolo di strumenti, o di vittime, dell'ambizione maschile ${ }^{1}$. 
Unico della cantica a toccare due bolge invece che una sola, il XVIII trae la sua peculiarità anche dal fatto di essere un canto di apertura, che con la descrizione dell'ottavo cerchio inaugura la seconda parte dell'inferno, quella dedicata alla frode, tra Malebolge e Cocito ${ }^{2}$.

2 Non è un caso che i commentatori moderni si siano dedicati particolarmente alla lingua di questo canto, che oscilla tra l'osceno della prima parte e lo scatologico della seconda ${ }^{3}$, e - pure in un medesimo registro basso - presenta uno stile differenziato come richiesto dai due tipi di fraudolenti presentati, i ruffiani e i seduttori della prima bolgia, gli adulatori della seconda ${ }^{4}$.

3 In apertura Dante descrive Malebolge, non solo un luogo ma un «ordigno» (v. 6), uno spazio che si fa strumento di pena e stanza della tortura, insieme naturale - la roccia che forma le bolge - e artificiale - i ponti che attraversano le bolge stesse -, e che talvolta sembra animarsi come un organismo vivente, per opprimere e non solo contenere i dannati, ma quasi stritolarli e azzannarli («assanna», v. 99) come un enorme mostro ${ }^{5}$ I dannati di entrambe le schiere cercano di sottrarsi allo sguardo del pellegrino, di non farsi riconoscere: ma Dante li mette alla berlina, li chiama per nome e cognome e così li denuncia ai lettori presenti e futuri, perché non ci sia ombra di dubbio sulla loro realtà anagrafica e sulla consistenza del loro peccato. Infamante è anche la pena, in un processo di progressiva degradazione fisica e morale che, dall'inizio alla fine del canto, attraversa le due bolge e ne presenta i prigionieri: se i ruffiani e i seduttori sono continuamente frustati da demoni cornuti che li battono «crudelmente di retro» e fanno loro, come si direbbe oggi, alzare i tacchi («levar le berze», v. 37), in un disperato tentativo di sottrarsi alla tortura, gli adulatori sono immersi nel proprio sterco, e continuamente si alzano o si accucciano nell'urgenza di defecare, col capo «di merda lordo» (v. 116) mentre si sforzano inutilmente di ripulirsi con le unghie ${ }^{6}$.

4 Il realistico linguaggio dantesco, giustamente definito «inesorabile» da Tiziano Zanato, dà così inizio alla seconda metà dell'inferno e "al regno della frode» ${ }^{7}$. La dominante stilistica del canto è quella di un registro basso, che comprende, come ha scritto Vittorio Celotto, «una selezione lessicale orientata verso il popolare, caratterizzata da numerosi volgarismi e inserti alloglotti, e associata a una tendenza alla rappresentazione realistica, perché focalizzata sui dettagli più corposi, di situazioni e personaggi umili o plebei», dove Dante non rifugge dall'uso di termini come «merda» appunto, o "puttana», secondo la regola che prescrive la «convenientia di linguaggio e contenuto» ${ }^{8}$.

5 Il canto è costruito poi sul parallelismo, come già osservava Lanfranco Caretti, anche per quanto riguarda la distribuzione dei peccatori: due moderni - Venedico Caccianemico fra i ruffiani e Alessio Interminelli fra gli adulatori - e due antichi o letterari, il seduttore Giasone per i primi e la prostituta Taide per i secondi; i moderni sono incontrati per caso e tutti due riconosciuti da Dante, gli antichi presentati da Virgilio e indicati come modelli; i due episodi si concludono entrambi con un invito ad andare oltre da parte della guida, senza attardarsi nella contemplazione dell'abbruttimento cui sono sottoposti i dannati («e questo basti de la prima valle», v. 98; «E quinci sien le nostre viste sazie», v. 136) ${ }^{9}$. Tuttavia, come osserva ancora Zanato, la parte dedicata a ruffiani e seduttori occupa ottanta versi, mentre quella incentrata sugli adulatori ne copre solo trentasei: segno questo della maggiore ampiezza dedicata da Dante alla prima bolgia, e della centralità del primo personaggio 
incontrato - il ruffiano Venedico Caccianemico - sul quale dovrà appuntarsi anche la nostra attenzione ${ }^{10}$.

Di Venedico - o Venetico - Caccianemico possediamo infatti molta documentazione, dalla quale si ricava il profilo di un uomo violento, nato intorno al 1228 da una famiglia bolognese di parte guelfa - schierata quindi con i Geremei e contro i Lambertazzi -: con la sua parte politica l'uomo svolge un'intensa attività per la quale finisce tre volte in esilio, ma nel 1274 i ghibellini vengono cacciati dalla città con l'aiuto dei marchesi di Ferrara $^{11}$. Venedico comincia così un'accorta politica matrimoniale, grazie al matrimonio del figlio Lambertino con Costanza, figlia di Azzo VIII d'Este; Dante invece lo accusa di essere un ruffiano e di avere prostituito la sorella Ghisolabella, spingendola «a far la voglia del marchese» (v. 56), ossia a cedere a Obizzo II d'Este, o secondo i commentatori antichi anche ad Azzo VIII, suo figlio e successore ${ }^{12}$. Di Ghisolabella si sa unicamente che era sposata con Niccolò Fontana, un ferrarese appartenente a una famiglia esiliata dalla città, anche se filoestense: tanto che alcuni ritengono che il Fontana sia stato esiliato dagli Estensi per questioni connesse proprio alla relazione della moglie col marchese ${ }^{13}$. Di Venedico Caccianemico abbiamo anche il testamento, datato 28 gennaio 1303, il che costituisce un piccolo problema: o Dante fa un errore, perché non ricorda l'anno di morte del peccatore e lo colloca all'inferno già nel 1300, oppure lo mette fra i dannati con un atto di volontaria e consapevole malizia ${ }^{14}$. È plausibile in ogni caso che il poeta conosca l'uomo politico durante il soggiorno bolognese del 1286/87; d'altra parte «l'episodio infernale, fortemente polemico con Bologna che fu ostile ai Bianchi fiorentini esuli, pare finalizzato in particolare a bollare come meretricio la politica matrimoniale dei Caccianemico e degli Estensi, tra i quali, poco dopo le nozze di Lambertino e Costanza, seguirono quelle di Fresco, figlio illegittimo ma erede di Azzo VIII, con Pellegrina, nipote di Venedico» ${ }^{15}$.

Il quadro storico, insomma, ci restituisce l'immagine di un mondo feroce, senza risparmio di colpi, dove le donne sono usate semplicemente come pedine per realizzare matrimoni economicamente e politicamente vantaggiosi o, peggio, per ingraziarsi i potenti inducendo addirittura le componenti della propria famiglia a «fare la voglia» di chi le desideri occasionalmente, di fatto costringendole a prostituirsi. Da qui la scena più lunga del canto - una scena veramente teatrale, come non si è mancato di osservare $^{16}$-, in cui prima Dante identifica il peccatore bolognese perché ne riconosce le fattezze («fazion», v. 49), poi il dannato risponde confermando la «sconcia novella» che lo riguarda - cioè la voce che proprio lui, per tornaconto, abbia convinto la sorella a cedere al marchese d'Este -; c'è quindi la delazione di Venedico, che si sofferma per svelare a Dante la presenza di molti bolognesi, puniti in quella stessa bolgia per il suo stesso peccato, ossia per avidità («rècati a mente il nostro avaro seno», v. 63); infine viene pronunciata l'apostrofe del diavolo, armato di una frusta di cuoio («scuriada», v. 64), che colpisce violentemente il ruffiano costringendolo ad andarsene ${ }^{17}$ :

E quel frustato celar si credette

bassando 'l viso; ma poco li valse,

ch'io dissi: «O tu che l'occhio a terra gette,

se le fazion che porti non son false,

Venedico se' tu Caccianemico.

Ma che ti mena a sì pungenti salse?».

Ed elli a me: «Mal volentier lo dico;

ma sforzami la tua chiara favella,

che mi fa sovvenir del mondo antico.

I' fui colui che la Ghisolabella 
condussi a far la voglia del marchese,

come che suoni la sconcia novella.

E non pur io qui piango bolognese;

anzi n'è questo luogo tanto pieno,

che tante lingue non son ora apprese

a dicer 'sipa' tra Sàvena e Reno;

e se di ciò vuoi fede o testimonio,

rècati a mente il nostro avaro seno».

Così parlando il percosse un demonio

de la sua scuriada, e disse: «Via,

ruffian! qui non son femmine da conio».

Il diavolo scaccia Venedico con gesti e parole di scherno, con la violenza fisica e con quella verbale, e con un'apostrofe che qualifica in maniera definitiva il Caccianemico come «ruffiano», cui si aggiunge la battuta sarcastica: «qui non son femmine da conio» (v. 66). Ora, sul significato ultimo di questa espressione c'è un generale accordo - qui non ci sono donne da prostituire -; molto meno sul senso letterale, sul quale si sono interrogate generazioni di critici.

9 Anna Maria Chiavacci Leonardi, pur non prendendo posizione su questo punto specifico, nel suo commento sottolinea il carattere violento della frase del demonio, leggendovi un attacco rude, espresso con linguaggio sprezzante, che liquida il dannato e lo bolla in maniera definitiva ${ }^{18}$ :

ruffian!: all'inizio del verso, la parola fin qui taciuta esplode con violenza, come una sferzata, che colpisce Venedico quanto quella materiale con cui si accompagna. - da conio: da moneta, cioè da prostituire per guadagno (così Jacopo, Lana, Benvenuto, Landino e molti). Altri intendono: da ingannare, prendendo conio nel senso di inganno (coniare per «ingannare» era di uso comune nel toscano antico). Tuttavia negli altri due luoghi del poema in cui si ritrova conio è riferito alla moneta (XXX, 115 e Par. XXIX, 126), e soprattutto il violento tono di spregio, e la frase finale della parlata precedente (v. 63), che fa centro sulla brama di denaro, vera causa di questa colpa, fanno scegliere il primo significato. Con questo durissimo verso Dante lascia Venedico, bollato per sempre, senza aggiungere altre parole.

10 Una tappa fondamentale del dibattito contemporaneo viene segnata dalla presa di posizione autorevole di Franca Brambilla Ageno, che imprime una svolta alla discussione schierandosi decisamente a favore dell'interpretazione di «conio» come 'inganno', proposta già nel 1875 da Isidoro Del Lungo a partire dagli statuti trecenteschi, e da un'interpretazione del verso dantesco messa in circolo già nella prima metà del secolo dall'Ottimo commento («coniare è mutare d'una forma in altra forma, e viene a dire ingannare, fare falso conio, falsa forma: trae il nome dalla moneta che piglia stampa») $)^{19}$.

11 Tuttavia a fine Ottocento, come la Ageno non manca di notare, Giuseppe Rigutini polemizza immediatamente con Del Lungo, e propone l'interpretazione dell'espressione dantesca come «femmine da moneta», trasformando così una contiguità semantica - quella del conio con la moneta da esso coniata - in una sovrapposizione perfetta ${ }^{20}$.

12 È interessante notare però che il grande Leo Spitzer, negli anni Venti del Novecento, ripropone un'ipotesi formulata già nel 1879 da Ludovico Passerini, che attribuisce all'espressione un significato osceno, dove il conio che batte la moneta diventa metafora per il membro virile, e l'atto di coniare allude a un rapporto sessuale violento $^{21}$. Questa stessa interpretazione, rimasta isolata in Italia, viene ripresa da Conner nel $1955^{22}$, ma la Ageno la scarta senza motivazioni di tipo interno al testo, limitandosi a classificare il ragionamento di Spitzer come un esempio «di quel 
deplorevole fenomeno per cui uno studioso, anche serio, si mette a interpretare un autore difficile come Dante col solo corredo della lingua contemporanea, non a Dante, ma allo studioso stesso» ${ }^{23}$.

Naturalmente il problema sta proprio qui, e non sfugge a una studiosa dell'acutezza di Franca Ageno: si tratta insomma di trovare testimonianze della metafora oscena nel volgare del Trecento; e d'altra parte le testimonianze addotte dai critici per istituire il parallelo conio-inganno, come si vedrà, non reggono ad un'analisi approfondita. Sta di fatto che il saggio della Ageno mette per lungo tempo una pietra sopra le altre interpretazioni, e per decenni l'equivalenza tra «coniare» e «ingannare» viene accettata da diversi critici italiani.

Vediamo allora su quali prove la Ageno fondi la sua dimostrazione, e su quali appigli di ordine storico-linguistico. Innanzitutto la linguista chiama in causa Alfredo Schiaffini che, recensendo Spitzer, propende per il significato di «coniare» come 'ingannare', perché lo spostamento semantico da coniare a ingannare «sarà avvenuto nel gergo dei falsificatori di moneta, che coniavano appunto per ingannare, donde si sarà poi diffuso, con ulteriore generalizzazione del senso... nella lingua comune» ${ }^{24}$. Come si vede, il ragionamento di Alfredo Schiaffini si basa su una pura ipotesi ricostruttiva («sarà avvenuto [...] si sarà poi diffuso»), della quale non c'è alcuna testimonianza scritta. In aggiunta, Franca Ageno cita un cantare trecentesco del senese Neri Pagliaresi, dove nel verso "pien di malizia e d'ogni falso conio» (riferito al diavolo) l'espressione "pien [...] d'ogni falso conio» significa ingannevole, come si deduce dal componimento stesso ${ }^{25}$.

Tuttavia l'espressione non può essere considerata una prova dell'uso di «conio» come 'inganno', nel testo di Dante e nella tradizione successiva. "Falso conio» infatti è binomio che definisce l'attività del falsario, di colui che inganna mettendo in circolo delle monete prive di valore perché imitazione delle monete ufficiali, quelle che escono dalla zecca. «Pien d'ogni falso conio» quindi non può che voler dire «ingannevole», non in grazia del fatto che l'espressione contiene il sostantivo "conio», ma perché contiene l'aggettivo «falso», che la connota in senso negativo.

Vediamo allora se si possono ricavare informazioni dall'uso del termine "conio» nella Commedia stessa, e dalle interpretazioni dei commentatori antichi, più vicini alla lingua del poema. Dante nella Commedia usa cinque volte il termine «conio» e sempre in senso proprio, con il significato letterale di 'stampo' o di 'sigillo', e mai nel senso di 'moneta': ma vediamo brevemente le occorrenze del sostantivo, tralasciando per ora il passo in causa.

In Inf. XXX Dante incontra il falsario di moneta Maestro Adamo - su cui si ritornerà nella seconda parte di questo saggio - e assiste alla lite con un altro dannato, il greco Sinone, reo a sua volta di una falsificazione di parola e non di moneta (ha fatto entrare con l'inganno il cavallo di legno a Troia, provocando la caduta della città ${ }^{26}$. Sinone apostrofa Adamo, ora impedito nel movimento degli arti perché appesantito dalla ritenzione idrica - dall'idropsia cui è condannato - e gli rinfaccia di avere avuto il braccio veloce quando era in vita, perché pronto a ingannare battendo più volte il conio sulle monete false che ha messo in circolo, mentre non si è mosso con altrettanta velocità quando si è diretto verso il rogo, dopo la condanna a morte («Quando tu andavi / al fuoco, non l'avei tu così presto [il braccio, n.d.r.]; / ma sì più e più l'avei quando coniavi», Inf. XXX, 109-111). E sotto Sinone aggiunge: «S'io dissi falso, e tu falsasti il conio» (v. 115): in entrambi i casi, «coniavi» e «conio» non si riferiscono alla 
moneta, ma all'atto di batterla e allo strumento con cui la moneta viene battuta, quindi Dante non confonde i due campi semantici.

Ancora in Par. XIX, 141 e XXIV, 87, il poeta mostra di distinguere perfettamente «conio» da «moneta», e di usare il termine per intendere lo strumento con cui si imprimono le immagini sul pezzo di metallo, anche quando lo impiega in senso figurato. È il caso di Par. XXIV, dove san Pietro saggia la fede del pellegrino con una metafora monetaria (vv. 83-87): «Assai bene è trascorsa / d'esta moneta già la lega e 'l peso / ma dimmi se tu l'hai ne la tua borsa»; e Dante risponde: «Sì ho, sì lucida e sì tonda, / che nel suo conio nulla mi s'inforsa». Infine nel canto XXIX del Paradiso Dante scrive il verso "pagando di moneta senza conio» (v. 126), per indicare il pagamento fatto con monete prive della garanzia del conio ufficiale, e quindi di nullo valore: se ne deduce che "conio» nella Commedia non sta mai per «moneta», ma accompagna il sostantivo «moneta», a dimostrazione del fatto che Dante ha piena consapevolezza della contiguità semantica $\mathrm{e}$ della differenza fra i due termini, che non sono sovrapponibili perché non sinonimi.

Sono stati semmai i commentatori a mettere in circolo l'equazione "conio» 'moneta', interpretando come "donne da moneta» l'espressione dantesca «femmine da conio» di Inf. XVIII: tuttavia, come si è appena visto, la lettura è smentita dall'uso che Dante stesso fa di «conio» nel resto della Commedia. Ma cosa dicono in proposito i commenti antichi? Nell'ultimo quarto del XIV secolo, solo Guglielmo Maramauro sovrappone i due termini, anche se in realtà preliminarmente interpreta "conio» in Inf. XVIII come un nome proprio, e commenta: «Questo Conio è uno castello in Romagna, el qual è fornito de meretrice assai. Altri dicono che questo se intende femena da dinari, idest che se dà altrui per dinari» ${ }^{27}$. Dunque Maramauro prende atto della difficoltà di interpretare l'espressione già alla fine del secolo; il suo commento è peraltro l'unico in cui si proponga una identificazione con l'omonimo borgo romagnolo, cui si attribuisce la pratica diffusa della prostituzione (evidentemente a partire proprio dal testo di Dante); dubitando della lettura, Maramauro suggerisce come seconda possibilità l'equazione «conio» 'moneta', e la attribuisce a non meglio precisati commentatori (è oggi nota la dipendenza del suo commento da quello fiorentino, molto precoce e risalente agli anni Trenta del Trecento, dell'Ottimo). Su conio/moneta torna anche Benvenuto da Imola: «e disse: via ruffian, quasi dicat: recede hinc in mala hora, non dicas plus de ista materia, quia nihil posses lucrari hic nisi verbera, quia: qui non son femine da conio, idest ad lucrum, ad denarios, ad pecuniam» ${ }^{28}$.

Tornando all'Ottimo, questi spiega invece: «dice femine da conio cioè da essere coniate et ingannate. Et in ciò nota che le femine sono mobile et abile ad essere ingannate» ${ }^{29}$. Una cinquantina di anni dopo, e indipendentemente, Francesco da Buti scrive pressoché le stesse parole: «qui non son femine da conio; cioè da essere coniate et ingannate con le tue seduzioni, che tu ti debbi restare a parlar con loro; e così li rimpruovera lo suo vizio» ${ }^{30}$.

21 Come dobbiamo intendere quel «coniate et ingannate»? Come una dittologia sinonimica, che ci autorizzi a riprendere in considerazione la lettura di Franca Brambilla Ageno? Ma la congiunzione «e» letteralmente coordina, dunque affianca il verbo «coniare» a «ingannare» e non ci autorizza alla sovrapposizione, ma semmai a una giustapposizione dei due concetti, coniare $e$ ingannare appunto, che evidentemente non sono la stessa cosa per i commentatori antichi... la congiunzione fa pensare semmai che si tratti di due azioni, due insulti cui sono sottoposte le donne; "coniate e ingannate» si potrebbe quindi leggere come: 'coniate quindi ingannate', 'coniate e poi 
ingannate'. Ma quale che sia il senso delle parole di Francesco da Buti, il suo commento non ci autorizza, lo ripetiamo, a concludere con sicurezza che "coniare» e «ingannare» siano sinonimi.

Molto interessante è poi l'Anonimo Fiorentino, perché ci restituisce la leggenda di Venedico Caccianemico, ormai consolidata ai primi del ' 400 e nata proprio sulla base dei versi danteschi ${ }^{31}$ :

Fu costui messer Venedico de' Caccianimici da Bologna; et fu provigionato uno tempo del marchese Azzo da Esti, signore di Ferrara. Avea messer Venedico una sua sorella, bellissima donna, detta madonna Ghisola, et antonomastice, per eccellenzia, però che avanzava in bellezza tutte le donne bolognesi a quello tempo, fu chiamata la Ghisola bella. Il marchese Azzo, udendo parlare della bellezza di costei, et avendola alcuna volta veduta per l'amistà di messer Venedico, ultimamente, sotto questa fidanza, si partì da Ferrara sconosciuto, et una sera di notte picchiò all'uscio di messer Venedico: messer Venedico si maravigliò, et disse che la sua venuta non potea essere senza gran fatto. Il Marchese, sotto gran fidanza, et perchè conoscea l'animo di messer Venedico, gli disse ch'egli volea meglio alla sua sirocchia, a madonna Ghisola, che a tutto il mondo; et ch'egli sapea ch'ell'era in quella casa: et pertanto, dopo molti prieghi, messer Venedico consentì et discese alla volontà del Marchese: partissi della casa, et lasciò lui dentro; onde il Marchese, giunto a costei, doppo alcuna contesa, ebbe a fare di lei; onde poi in processo di tempo la novella si sparse: et perché parea forte a credere che messer Venedico avesse consentito questo della sirocchia, chi dicea la novella et apponevala a uno, et chi a un altro; di che ora messer Venedico chiarisce a Dante, et dice che, come che questa novella si dica, io fui quelli che condussi costei a fare la volontà del Marchese.

La storia diventa così una novella di stampo decameroniano, con il marchese d'Este che bussa alla porta dei Caccianemico di notte in gran segreto, cercando di non essere visto, e svela a Venedico la propria ossessione erotica per la sorella di lui, Ghisolabella; il bolognese che cede alle pressioni del signore di Ferrara, come un vassallo che debba accondiscendere ai capricci del feudatario, e accetta di uscire di casa per lasciare campo libero all'Este... solo a questo punto il marchese raggiunge la Ghisolabella, con la complicità di Venedico che si è allontanato per non doverla difendere, e finalmente riesce a congiungersi carnalmente con la donna, non senza resistenza da parte di lei («doppo alcuna contesa»). La novella della Ghisolabella e del marchese, così come prende forma sotto la penna del commentatore fiorentino, non è quindi la storia di una consenziente - seppure colpevole - relazione adulterina, ma quella di uno stupro bello e buono, reso possibile dal comportamento di Venedico Caccianemico, per il quale Dante pronuncia la condanna all'inferno.

Quanto all'espressione «femmine da conio», era probabilmente molto chiara all'epoca, se Guiniforte Barzizza, compilando nel primo Quattrocento il suo commento per i Visconti, non sentiva neanche il bisogno di spiegarla, ma semmai di scagionare il popolo bolognese dalle accuse con cui Venedico lo aveva bollato: «Così parlando costui un Dimonio lo percosse con la scuriada e disse: "Via, ruffian! qui non son femmine da conio". Questa è la sentenza del testo. Tacere non posso. Io non sono Bolognese, ma onestà mi costringe a parlare. Mal fa chi fuori di storia ad alcuna particolar persona infamia porta, peggio chi ad un lignaggio; ma pessimamente chi ad un popolo, ed in ispecialità, che sia generoso e magnanimo» ${ }^{32}$.

Dai commentatori antichi non si ricava quindi né un'interpretazione univoca, né una parafrasi chiara del testo. Se la nostra ipotesi che si tratti di una metafora oscena è corretta, la questione è allora: perché i commentatori la hanno elusa? Perché non 
sciogliere la metafora, dandone il significato letterale invece di offrirne solo l'interpretazione, e così scartando il primo termine del paragone implicito nell'immagine, e limitandosi ad enunciare il secondo?

Forse la metafora era fin troppo conosciuta, e non c'era nessun bisogno di spiegarla ai lettori tre e quattrocenteschi - ma in questo caso dovremmo dimostrarlo, trovandone testimonianza -; oppure si trattava di un'immagine oscena che creava disagio, era talmente forte da mettere in imbarazzo gli stessi commentatori, rispetto ai quali Dante avrebbe mostrato una volta di più l'oltranza linguistica di un realismo senza pari.

Prendiamo innanzitutto in esame il secondo assunto, ossia l'eventualità che i commentatori antichi si siano trovati in difficoltà con quella che gli esegeti moderni chiamano, dopo Edoardo Sanguineti, la «visiva aggressività» di Dante in Malebolge, il suo linguaggio duro, plebeo, che nel descrivere l'inferno non rifugge da nessuna delle possibilità offerte dal lessico e dalla sintassi volgari ${ }^{33}$.

Zygmunt Baranski definisce icasticamente il canto un «impasto di sesso e di escrementi $»^{34}$ - un canto "scabroso», come lo ha giustamente definito Tiziano Zanato $^{35}$-, che ha dato problemi ai commentatori antichi e a quelli moderni. Appoggiandosi sul commento di Francesco da Buti, Baranski osserva come Inferno XVIII, particolarmente nell'ultima parte, sollevi «notevoli problemi di convenientia poetica» per un lettore medievale ${ }^{36}$. Il Buti infatti, citando Orazio, rimprovera a Dante «l'arditezza della presentazione, pur sentendo il dovere, da bravo cultore dell'Alighieri, di attenuare i toni di biasimo» ${ }^{37}$. Baranski, cogliendo l'imbarazzo del commentatore trecentesco, non può esimersi dal rilevare una ovvia «dimensione sessuale» della prima bolgia, e dal cogliere invece nella seconda "eccessi di sesso ed escrementi» ${ }^{38}$ : è l'ambito di ciò che oggi chiamiamo rispettivamente "osceno» e «scatologico», cui anche la cultura medievale dava voce ma in modi diversi per l'uno e l'altro aspetto ${ }^{39}$. E se la maggior parte dei commentatori attuali ritiene che Dante scelga un linguaggio scabroso e foneticamente aspro, nonché «torture nauseanti come segno del suo profondo spregio verso colpe e peccatori che egli considera specialmente disgustosi» $»^{40}$, Baranski mette in luce il retroterra biblico celato dietro al canto e al suo linguaggio, un retroterra che fra i primi commentatori solo Graziolo Bambaglioli e Pietro Alighieri mostrano di avere compreso, e che ci dà ragione di scelte formali e contenutistiche non riconducibili al solo disprezzo verso i dannati delle prime due bolge ${ }^{41}$.

29 L'«arditezza linguistica» dell'Alighieri deriva insomma dallo stile biblico, dove si fa spesso ricorso a un linguaggio di tipo scatologico, dove gli escrementi e il «basso corporeo» di bachtiniana memoria sono un elemento ricorrente ${ }^{42}$ : è nel «pluristilismo totalizzante del sermo humilis scritturale» che Dante trova un modello per il linguaggio con cui descrive le pene dei ruffiani e degli adulatori, e tuttavia il modello biblico dà ragione soprattutto del versante scatologico, non delle allusioni oscene ${ }^{43}$.

La Bibbia infatti, e la cultura cristiana medievale con essa, mostrano a Dante una «relativa disinvoltura nell'uso della terminologia scatologica», e invece una «scrupolosa discrezione verbale nel presentare soggetti carnali», come Baranski non manca di osservare $^{44}$. L'osceno viene condannato sia dalla precettistica cristiana sia da quella letteraria, fa parte del turpiloquium e dunque di un «peccato della lingua» di cui il fedele non può macchiarsi $\mathrm{i}^{45}$ : da qui una maggiore «reticenza linguistica di Dante tra $\mathrm{i}$ peccatori sessuali» e, aggiungiamo noi, l'opportunità del ricorso a metafore quando si entra nella sfera del sesso, per la quale la cultura medievale nutre un imbarazzo insopprimibile ${ }^{46}$. Questo può spiegare anche la «reticenza» dei commentatori antichi, 
ossia la tendenza a mettere in ombra, se non a scartare del tutto, la metafora erotica insita nell'espressione «femmine da conio».

Ma veniamo ora al primo dei due assunti da cui siamo partiti per dimostrare la validità della nostra interpretazione, ossia all'idea che la metafora del conio per indicare l'atto sessuale fosse fin troppo conosciuta, e non ci fosse nessun bisogno di spiegarla, né da parte di Dante né da parte dei suoi commentatori che, trovandosi in imbarazzo, ne avrebbero eluso il significato letterale, proponendo ai lettori spiegazioni per così dire 'addomesticate', in cui il senso di fondo rimane, ma il realismo crudo dell'espressione viene totalmente disinnescato («femmine da conio» viene spiegato ora come 'donne coniate e ingannate', ora come 'donne da vendere a prezzo').

La validità di questo assunto, abbiamo detto sopra, va però dimostrata, trovandone almeno una documentazione, una testimonianza nella letteratura del tempo. Spiegando infatti il v. 66 di Inf. XVIII, Saverio Bellomo commenta:

Se conio, cioè il punzone che imprime l'immagine della moneta, è metonimia per 'denaro', l'espressione vale 'donne da prostituire', da collegare all'avarizia chiamata in causa al verso 63; oppure conio è da coniare 'ingannare' e coniello 'inganno' (cfr. Statuti senesi, dist.5, cap. 35), quindi 'donne da ingannare' (cfr. Ageno, Studi, pp.9-12). L'ipotesi che sia metafora oscena, giacché il punzone può alludere al membro virile, è plausibile e potrebbe convivere in chiave anfibologica con il primo significato, ma non è confortata dai primi commenti né da documentazione ${ }^{47}$.

Tuttavia, se i commenti non ci aiutano, le prove documentarie - che in passato erano difficili da scovare nella mole dei manoscritti e dei testi a stampa - sono più facili da individuare oggi, quando molti dei testi della nostra tradizione letteraria sono digitalizzati o almeno indicizzati in rete: e noi riteniamo di averle trovate.

La prima è un passo ambiguo del Ristorato, un poemetto in terzine a rima incatenata - elaborazione poetica del Fiore di Virtù con reminiscenze dantesche - composto negli anni Sessanta del Trecento dal fiorentino Ristoro Canigiani ${ }^{48}$. Nel capitolo XXXIX, dedicato alla lussuria contrapposta alla castità, Ristoro spiega che il vizio

Discende propiamente e viene in quegli,

Che si congiungon sanza matrimonio

Aver contratto, o preso pe' capegli.

E questo disonesto e brutto conio,

Fornicazion s'appella tra' mortali,

La qual vien per fattura del demonio ${ }^{49}$.

Ristoro distingue poi dalla fornicazione l'adulterio, perché commesso da chi è già sposato, quindi l'incesto, in quanto consumato fra parenti. Il passo è ricordato, proprio a proposito del termine conio, nel Tesoro della Lingua Italiana delle origini (TLIO), dove il sostantivo viene ricondotto dubitativamente al significato di 'modo', o 'abitudine', ma in ogni caso «in relazione alla lussuria» ${ }^{50}$.

Più esplicito, e dal significato assolutamente univoco, è un componimento successivo, ossia la Canzona de' tagliatori di legname, un testo apertamente osceno di Bernardo Angiolini, riconducibile alla tradizione carnascialesca del Rinascimento, dove si legge ${ }^{51}$ :

Consiste l'arte nostra in un sol punto:

nel dar gran colpi e buoni,

massime quando appresso il fin s'è giunto:

ch'allor non t'abbandoni,

ma tocchi e suoni

infinché giù ne vegni.

In duo colpi facciam quel, ch'altri in venti, 
che nollo fare' Marte,

con queste scure e con certi stormenti

che noi tegnàn da parte:

e questa è l'arte

degli alpestri regni.

Pigliate per vostr'uso il legno verde,

donne, ch'è buon per voi:

nel vecchio è poco vigore, onde si perde

il tempo, che duol poi;

e anche noi

vi facciam su disegni.

E buon colpi si dà, quando v'è fitto

il conio tutto quanto;

ma sopratutto vuol esser diritto,

sendovi fesso o stianto:

e meniàn tanto

ch'a forza apriàno i legni. [...]

Evidentemente la metafora era nota nei testi di tipo teatrale, nei quali era concesso l'uso di un linguaggio molto esplicito relativo alla sfera corporea, e anche a quella sessuale. Infatti ne troviamo ancora traccia nella commedia dell'arte seicentesca, più precisamente nel testo di una donna, la scrittrice - e cortigiana - Margherita Costa, che apre la sua commedia Li buffoni (1641) con un Prologo dalle battute salaci, atto a invogliare gli spettatori a guardare lo spettacolo, dove deridendo gli uomini che tradiscono la moglie, come il protagonista della commedia, si dice ${ }^{52}$ :

Dormir nel letto altrui con l'altrui donna?

Oh buono! E poi voler dare ad intendere

Ch'ei 'l fa per risparmiare i suoi lenzuoli.

Gnaffe! Egli vuole che col conio suo,

Sol la moneta, ch'è di altri, si stampi;

E gode arare in licenzioso modo

Non già co' buoi, ma con la fronte i campi.

Questi testi sono solo tracce, singoli episodi, e certamente altri se ne possono trovare, ma confermano una presenza della metafora tra il tardo Medioevo e l'età moderna. E non è forse un caso che gli ultimi due appartengano al filone teatrale, perché il teatro è anche il sottotesto di Inferno XVIII, un canto dalla forte carica di teatralità, come da più parti è stato osservato ${ }^{53}$.

Il personaggio emblematico della seconda bolgia, la prostituta Taide, deriva proprio dal teatro, ossia dalla commedia terenziana Eunuchus, e viene presentata da Dante come caso esemplare di adulazione ${ }^{54}$. Dunque, come sottolinea Zanato, la donna è punita in quanto adulatrice più che come prostituta, e «in lei si colpiscono tutte le prostitute, considerate adulatrici di professione. Non si condanna dunque una puttana perché fa mercimonio del proprio corpo [...], ma perché inganna il maschio, lusingandolo» ${ }^{55}$.

Con i testi di Bernardo Angiolini e di Margherita Costa siamo cronologicamente lontani da Dante, ma gli esempi forniti sembrano suggerire una continuità, e confermare un uso popolare della metafora in quel registro basso che pertiene alla sfera "comica" e teatrale cui appartiene anche il canto inaugurale di Malebolge. Dante non solo utilizza un personaggio teatrale come Taide, ma soprattutto fa teatro nel canto dei ruffiani e degli adulatori, o meglio fa «le prove - come rileva Stefano Carrai - per quella brillante invenzione da dramma liturgico» che sarà l'episodio dei barattieri e dei Malebranche nella quinta bolgia ${ }^{56}$. 
41 Se Dante ha costruito nel XVIII versi dalla forte carica di teatralità (e non solo nella parte dialogica), perché non pensare che abbia usato una metafora molto chiara agli occhi dei suoi contemporanei, e circolante proprio negli ambienti e nei testi teatrali? I lettori del Trecento avevano ben presente come si coniava moneta e potevano stabilire un nesso tra la battitura della moneta stessa e un rapporto sessuale di tipo violento, perché non consensuale, neppure quando la donna accettava di soggiacere a matrimoni o a relazioni occasionali: ma vi soggiaceva perché in effettiva condizione di inferiorità sociale e psicologica, e perché all'epoca era molto frequente, da parte femminile, accettare questo tipo di imposizioni quando richieste dalla famiglia, per ragioni politiche o economiche. Pensiamo al terzo canto del Paradiso, dove Piccarda Donati racconta come sia stata strappata al monastero - cui inizialmente era stata destinata, probabilmente anche in questo caso per ragioni di opportunità - ad opera del fratello Corso, in quanto sviata verso un matrimonio vantaggioso per la potente famiglia dei Donati. Sottratta al convento e alla scelta di vita monastica che aveva ormai abbracciato, la Piccarda dantesca cala letteralmente un velo sul matrimonio infelice con il violento Rossellino della Tosa: «Iddio si sa poi qual mia vita fusi» (Par. III, 108), dice soltanto lo spirito, alludendo con pudore al destino a cui come donna non poteva opporsi ${ }^{57}$.

Le donne «coniate e ingannate» dell'Ottimo e del Buti subivano uno stesso destino, erano vittime dello stesso tipo di violenza domestica, e il poeta all'inferno colloca i loro carnefici giudicandoli con durezza estrema: per la prima volta, qui Dante ci appare «crudele» ${ }^{58}$; sceglie dei supplizi - osserva ancora Carrai - «che avvicinano alla condizione animale per coloro che si sono fatti sfruttatori e profittatori della altrui benevolenza» ${ }^{59}$.

43 Ed è sempre Carrai a sottolineare il parallelismo tra la chiusa della prima bolgia con le «femmine da conio», e della seconda con la «puttana» Taide ${ }^{60}$ : un parallelismo che non può sfuggire a una lettura attenta, e che riconduce tutto il canto a una riflessione sulla sessualità mercificata, utilizzata come fonte di guadagno o merce di scambio ${ }^{61}$. Subito dopo Venedico appare infatti Giasone, "prototipo letterario del seduttore» ${ }^{62}$ : ma «il Giasone chiamato qui in causa, più ancora del protagonista [...] delle Metamorfosi, è il deuteragonista delle Eroidi sesta e duodecima che Ovidio aveva immaginato gli fossero state indirizzate dalle sedotte e abbandonate Isifile e Medea» ${ }^{63}$. L'eroe lascia infatti Isifile incinta di due gemelli, giurando di tornare; parte poi per l'impresa del vello d'oro, per conquistare il quale seduce Medea, quindi abbandona anche la maga e ne subisce l'atroce vendetta sui figli. Subito dopo, tra i lusingatori, Dante riconosce Alessio Interminelli, appartenente a una nobile famiglia di parte bianca esiliata da Lucca ${ }^{64}$; tuttavia «il suo vizio non è documentato, e la notizia che "tenne bordello di puttane" - chiose Selmi - pare illazione suggerita dal testo dantesco»: i commentatori antichi, evidentemente, avvertivano già che il tema della prostituzione serpeggia per tutto il canto, a prescindere dai peccati condannati nelle due bolge, e anzi rappresenta - per dire così - il denominatore comune a entrambe le bolge e a tutti i personaggi, dal ruffiano bolognese alle femmine da conio, e dal lusingatore lucchese a Taide ${ }^{65}$.

Peraltro il canto, come sottolinea ancora Zanato, è pervaso di metafore alimentari («non sono digiuno», v. 42; «pungenti salse», v. 51; «asanna», v. 99; «s'apasta», v. 107; «gordo», v. 118; «stucca», v. 126; "viste sazie», v. 136, peraltro evidenziate in fine di verso e corrispondenti alla rima) ${ }^{66}$, che sono tipiche della Commedia ma non solo: «si insinua il sospetto che possa sussistere un sotterraneo legame fra la frequenza inusuale 
di tali tropi e uno dei temi portanti del canto, il sesso (presente anche nella seconda parte, con Taide la puttana) e certo non serve scomodare Freud per sottolineare il rapporto esistente fra cibo ed eros, se è vero che Dante stesso ne era conscio, almeno a leggere i verso 32-33 della canzone Poscia ch'amor del tutto m'ha lasciato»" ${ }^{67}$.

Dunque un «tema portante» sta al fondo del canto, e non è quello della frode, dell'inganno - che riguarda tutte le Malebolge - ma quello del sesso sfruttato e mercificato, al quale rimanda anche l'espressione «femmine da conio» con cui il diavolo, e Dante alle sue spalle, liquida violentemente il guelfo bolognese. Lo stesso Zanato - che propende per l'interpretazione «da moneta, da sfruttare» - suggerisce che

[...] come ulteriore significato, una volta scartato conio 'inganno', messo in circolo dall'Ottimo, non foss'altro perché sarebbe tautologico e vicino al grado zero di senso in un cerchio di dieci bolge in cui tutti sono fraudolenti, andrà considerato il valore fallico della parola, partendo dal 'punzone' [...], e che pare non inopportuno in una bolgia che diremmo, quantomeno, maschilista. Di conseguenza, qui non son femmine da conio si può rendere - con le parole di Pagliaro - "'non ci sono femmine su cui batter moneta', ed è da intendere in un senso più vasto che non il solo guadagno di denaro" $\rangle^{68}$.

Anche Ezio Raimondi, richiamato da Zanato nel passo appena citato come il critico che più «ha insistito fra gli altri, con signorile discrezione» per questa lettura ${ }^{69}$, segue la linea dei linguisti che da Spitzer a Pagliaro hanno sostenuto l'interpretazione di «femmine da conio» come metafora oscena, sulla base dei criteri interni al canto che abbiamo qui cercato di mettere in luce. A queste motivazioni abbiamo poi aggiunto la documentazione offerta da opere che, fra tardo Medioevo ed età moderna, testimoniano la diffusione della metafora in ambito letterario. Ora vedremo che cosa sia un conio al tempo di Dante, e quali immagini possa evocare nel pubblico dei lettori trecenteschi.

\section{Cos'è un conio}

Per cercare di capire il senso della frase di Dante è necessario conoscere i modi di produzione delle zecche, la tecnologia, e tutto ciò che la moneta metallica poteva rappresentare nel mondo di Dante. Vedremo quindi i termini conio e gli altri menzionati da Dante in relazione alla moneta, alla zecca, e il loro contesto fiorentino.

È utile cominciare leggendo la voce conio nel Vocabolario Treccani on-line ${ }^{70}$ :

cònio $^{2}$ s. m. [lat. cŭneus; cfr. cuneo]. - 1. Cuneo per spaccare legna. Anticam., sinon. Di cuneo anche nel sign. architettonico e militare. 2. a. Utensile per la lavorazione plastica dei metalli, consistente in un blocchetto di acciaio duro che porta in cavo disegni, iscrizioni e figure che possono essere impresse in rilievo sul pezzo lavorato; è fissato alla slitta di un bilanciere o di una pressa, da cui viene spinto sul pezzo metallico in modo che questo, sotto la stretta, riempia esattamente le cavità. b. L'impronta fatta col conio: contraffare il c.; le monete buone si conoscono al c.; batté una moneta falsa del c. fiorentino (Machiavelli). c. non com. La moneta stessa coniata. d. L'operazione e l'effetto del coniare: moneta di nuovo c. (o nuova di c.); fig., parola di nuovo c.; una storiella nuova di conio. e. fig. Specie, tipo, qualità: persona di basso, di ottimo c.; ho da fare con gente d'ogni c.; tre furfanti, tutti dello stesso conio. f. Con senso chiaramente dispregiativo, ma sulla cui interpretazione i commentatori sono in disaccordo, nel verso dantesco qui non son femmine da conio (Inf. XVIII, 66), per lo più intesa come «non vi sono femmine da prostituire, o che si prostituiscono, per denaro» oppure «femmine da ingannare, da sedurre con arti fraudolente». 
Nei significati 1 e 2 della voce cònio, dal latino cŭneus, il conio è strumento che imprime, anche con forza (spazzare la legna; incidere in cavo), lasciando una impronta indelebile. Con il significato 2.c conio è usato in modo errato come sinonimo di moneta, senza pensare che si tratta di due manufatti diversi, il primo genera il secondo; questo uso improprio del termine è in genere conseguenza dell'insegnamento sui banchi di scuola, quando l'insegnante invita (o invitava?) a non usare sempre le stesse parole. In realtà se si scrive di monete si deve usare 'monete', oppure fiorini, ducati, bolognini, ecc., e si può dire eventualmente 'monete di conii diversi' per indicare monete di diverse emissioni o tipi della stessa zecca. Lo studio dei conii in monete di uno stesso tipo implica l'esame attento di ogni dettaglio delle immagini e delle iscrizioni: si tratta di un esame tecnico proprio della ricerca numismatica che permette di riconoscere se due monete sono state prodotte dagli stessi conii, o dallo stesso conio per un lato e due conii diversi per l'altro; trovare monete di conio identico in un tesoro significa che esse furono tesaurizzate poco dopo essere state emesse dalla zecca senza venire disperse nella circolazione ${ }^{71}$.

Nello stesso Vocabolario la voce coniare è la seguente:

coniare v.tr. [der. di conio ${ }^{2}$ ] (io cònio, ecc.). - 1. Battere monete o medaglie facendovi l'impronta col conio. 2. fig. Inventare, creare, spec. vocaboli o locuzioni: è una parola coniata da me; èabilissimo nel c. soprannomi; igiornalisti coniano continuamente nuove parole; meno com. riferito ad altre cose: c.notizie false, c. fandonie; fra' Greci l'arte musicale ... non offriva ancora abbastanza al compositore da c.e inventar di pianta nuove melodie (Leopardi). Part. pass. coniato, anche agg., impresso col conio: monete coniate; tra le suddette medaglie antiche ... si vede coniato un Ercole (Redi).

51 Il conio dà l'impronta e quindi 'crea' monete e medaglie; metaforicamente crea parole nuove mai esistite prima. Si noti che le collezioni di conii e punzoni delle zecche sono definite «materiale creatore»; vi sono numerose collezioni di materiali delle zecche italiane preunitarie ancora esistenti. La sopravvivenza dei conii è rara e più frequenti sono quelli non ufficiali, abbandonati nella fretta. La zecca ufficiale invece, quando le impronte di un conio diventavano usurate, le ritirava, ne tagliava la superficie incisa, e la rilavorava; quando poi il conio diventava troppo corto veniva riutilizzato il metallo. Solo per Massa Marittima si conservano diversi conii medievali, forse archiviati a Siena dopo la conquista della città maremmana ${ }^{72}$. I conii erano strumenti preziosi e carichi di simbolismo ${ }^{73}$ con i quali si dava al metallo pesato quella impronta ufficiale che ne garantiva valore e bontà; il conio era dunque non solo immagine del sovrano o dello Stato, ma era una delle più importanti essenze dello Stato; per questo, e non solo per il danno economico, lo Stato puniva e punisce i falsari, e per questo i conii ufficiali erano controllati severamente ${ }^{74}$.

Leggendo la voce Conio di Eugenio Ragni nell' Enciclopedia Dantesca del 1970, in particolare in merito alle «femmine da conio», stupisce constatare la lontananza dei commentatori da quella che - secondo la ricerca su temi monetari medievali - doveva essere la reale conoscenza di Dante in fatto di zecca e monete: monete, d'oro, d'argento e di mistura della zecca di Firenze e non solo, che il poeta avrà maneggiato, valutato, verificato per il loro valore corrente, e anche per stare in guarda dalle imitazioni o falsi che pure circolavano nonostante le cure dello Stato.

Prima dunque di esaminare l'espressione «femmine da conio» è utile esaminare alcuni passi danteschi relativi alle monete. 
Dante fa riferimenti molto precisi alle monete, con indicazioni anche sui processi tecnologici della produzione. Per capire a pieno il significato dei suoi riferimenti si devono quindi conoscere le monete nella realtà fisica, a partire dai processi produttivi nella zecca.

mecca nella lingua italiana si riferisce sia all'istituzione che ha il diritto di battere moneta (una repubblica, un re, un duca: senza autorizzazione giuridica non vi è moneta ufficiale) e sia alla zecca come sede e officina, o molteplici sedi e officine di una zecca istituzione ${ }^{75}$. Come esempio di sedi diverse per una zecca ufficiale si veda il caso dei fiorentini che nel 1256 batterono "per dispetto» monete con i loro conii ufficiali sulle rive del Serchio (a San Jacopo in Val di Serchio) per celebrare la vittoria contro i pisani che avevano attaccato Lucca. La cronaca di Giovanni Villani ricorda che in questa occasione i fiorentini

[...] quivi tagliaro uno grande pino, e battero in sul ceppo del detto pino i fiorini d'oro; e per ricordanza, quegli che in quello luogo furono coniati ebbono per contrassegna tra' piedi di San Giovanni quasi come uno trefoglio, a guisa d'uno piccolo albero: e de' nostri dì ne vedemmo noi assai di quelli fiorini.

Anche nel 1363 i fiorentini festeggiarono nello stesso modo a Riglione la vittoria contro i pisani; i documenti della zecca registrano i pagamenti fatti per realizzare i nuovi conii con la volpe schiacciata dai piedi san Giovanni Battista («ferra nova cum signo et figura vulpis»), dove la volpe indicava Pisa (Dante parla di «volpi pisane»). Poco dopo nel 1363 i pisani ebbero una riscossa e a Rifredi batterono moneta con l'aquila che tiene il leone (guelfo) negli artigli ${ }^{76}$.

Questi episodi di coniazioni "per dispetto», come li definì Philip Grierson, ci conducono subito nell'aspetto simbolico del batter moneta, tramite il gesto di 'segnare', obliterare, marchiare e impregnare il territorio nemico con l'immagine sovrana dell'identità della città vittoriosa.

58 Le immagini non sono meno importanti della lega e del peso delle monete. Isidoro di Siviglia precisò che le tre caratteristiche delle monete - metallo, immagini e peso erano ciascuna fondamentale e imprescindibile: se solo una di queste è carente (metallo di lega non conforme, immagini improprie, peso irregolare), non è moneta ${ }^{77}$. Le monete fino a tempi relativamente recenti erano di vero oro e argento, di leghe diverse (e certamente lo erano al tempo di Dante): lega e peso dovevano rispettare le ordinanze statali. Chi usava le monete conosceva il significato di termini come 'carati' e 'once', con i quali si indicava il contenuto metallico ${ }^{78}$, e l'iconografia era anche ben nota, con variazioni che di volta in volta indicavano un valore diverso. Così, le monete di Firenze erano contrassegnate su un lato dal giglio e legenda +FLORENTIA, e sull'altro dal san Giovanni Battista e legenda +S IOHANNIS B; il santo poteva essere stante o seduto, con dettagli diversi delle vesti. I signori della zecca, inoltre, marcavano le monete con un simbolo ad essi corrispondente che dal $1303 \mathrm{fu}$ registrato nel grande libro detto Fiorinaio o Libro della Zecca nell'Archivio di Stato di Firenze. Ogni sei mesi si sostituivano i 'signori' responsabili della zecca per l'oro e di quella per l'argento, ad evitare conflitti di interesse.

Una zecca, intesa come sede operativa, era un complesso impianto di produzione industriale con precisa divisione del lavoro: per evitare furti anche dei più piccoli frammenti di metallo, si controllava con oculatezza il passaggio del metallo dall'officina del fonditore a quella dei tagliatori dei tondelli, e da questi a quella degli arrotondatori, e infine all'officina dei coniatori. I monetieri a Firenze erano organizzati in una sorta di 
corporazione, di cui ci resta lo statuto (Constitutum artis monetariorum civitatis Florentie) del 1310 circa; nessun monetiere poteva lasciare Firenze per lavorare in un'altra zecca senza un permesso speciale del prevosto; se un monetiere non fiorentino fosse arrivato a chiedere assistenza, l'associazione l'avrebbe aiutato a proprie spese, procurandogli il denaro necessario per tornare a casa: in altre parole, qualsiasi lavoratore non fiorentino veniva escluso. L'impressione che si ricava dal testo è quella di un eccellente codice di buon comportamento: i monetieri non lavoravano durante le festività religiose e nemmeno in caso di morte di uno di loro; non dovevano bestemmiare, né offendere i colleghi o criticare il lavoro prodotto da altri; era loro dovere aiutare i colleghi poveri o malati assistendoli con l'elemosina; contemporaneamente cercavano anche di prendersi cura dei propri interessi, rifiutando ogni diminuzione del salario e controllando l'accoglienza dei nuovi membri; tutto questo però non era sufficiente a proteggere la loro professione; la paga era molto bassa e non esistevano privilegi; siamo a conoscenza anche di alcuni scioperi e ribellioni ${ }^{79}$.

60 A Firenze la prima zecca ebbe sede in una casa con torre nell'area in cui fu poi costruita la Loggia dei Mercanti (oggi dei Lanzi). La zecca era una istituzione e realtà di impresa ben presente nella città. Come tutte le zecche nel medioevo lavorava non solo e non tanto con il metallo dello Stato, ma soprattutto con il metallo, monetato e non, portato da privati (mercanti, banchieri o altri) per ottenere quantitativi corrispettivi in moneta nuova. Quanto più le monete di una zecca erano pregiate e richieste anche al di fuori del territorio, tanto più vi era interesse a procurarsele, e anche a falsificarle.

61 Solo gli Stati possono battere moneta e quindi le monete sono emanazione sovrana per eccellenza: il delitto di falsa moneta fu considerato dalla tarda età imperiale romana un delitto di lesa maestà e punito con pene severissime fino alla morte. Il reato del falsario obliterava in certo modo l'autorità sovrana nel momento in cui la moneta falsa, per le false impronte su una lega cattiva, determinava il rifiuto della moneta stessa.

Dante ci porta quasi all'interno di una zecca tramite maestro Adamo nell'Inferno (XXX, 58-90). Qui il falsario condannato al rogo è definito «maestro Adamo» e alcuni studiosi hanno creduto che l'appellativo maestro presupponesse un titolo accademico ${ }^{80}$. Va detto invece che i responsabili di una zecca nel medioevo erano comunemente definiti 'maestri' ('magister' oppure 'dominus'), il che si riferiva al loro essere a capo della conduzione, con la sapienza necessaria per la gestione e la competenza in relazione alle varie fasi della produzione: lavorazione, conoscenza del metallo e delle leghe, e molto altro ancora. Vi sono in merito numerose fonti relative a diverse zecche italiane comunali che qui non esamino. La documentazione sulla zecca di Firenze è importante e non credo vi siano dubbi che Adamo fosse un 'maestro' per la sua competenza tecnica e operativa di zecca senza doverlo rintracciare in accademie. A Firenze i responsabili della zecca erano definiti 'signori' ed erano due, uno per l'oro ed uno per l'argento: le due produzioni dovevano essere distinte, quasi due zecche parallele, ciascuna con personale distinto. La contabilità, il passaggio di metallo pesato e registrato, erano distinte: tanto oro o argento entrava per la trasformazione in moneta nuova, tanto doveva uscire, fatto salvo il calo naturale dovuto alla fusione.

63 Dante fa dire ad Adamo di che tipo fosse precisamente la frode compiuta nella zecca voluta dai conti Guidi nel loro castello di Romena:

Ivi è Romena, là dov'io falsai

la lega suggellata del Batista;

$[\ldots]$ 
ei' m'indussero a batter li fiorini,

ch' avevan tre carati di mondiglia.

64 I fiorini d'oro di Firenze erano di oro puro, pari a 24 carati. Per conto dei Guidi, Adamo produceva falsi fiorini di Firenze a 21 carati invece che i 24 originali, aggiungendo ai 21 carati di oro "tre carati di mondiglia», vale a dire rame o lega bassa di argento. Si noti tra l'altro che per realizzare queste leghe era necessaria grande competenza nei calcoli per la fusione. Probabilmente l'impresa si sarà servita di un buon incisore orafo per realizzare i conii, e di bravi operai: la catena produttiva per realizzare monete false si basava su specifiche competenze, forse ancor più che in una zecca ufficiale. Per esempio il fiorino di oro puro doveva pesare 3,53 g; dovendo realizzare una moneta di 21 carati, si doveva risolvere il problema della riduzione di peso, avendo l'oro un peso specifico superiore a quello di altri metalli.

Il pericolo dei falsi era grandissimo. La lista di monete datata 1307 contenuta in un trattato di aritmetica conservato nella Biblioteca Marciana di Venezia si concludeva con questa frase: «Che tu non ti fidi a queste leghe, per che tuttavia si mutano le monette ed anche si falsano; però non te fidare si primamente ne chiarisci assagio di fuoco overo d'altro modo» ${ }^{81}$.

Nella realtà, comunque, non era facile per il pubblico saggiare le monete in circolazione, ed è così che monete false si trovavano normalmente tesaurizzate tra monete buone, senza che il proprietario se ne fosse accorto ${ }^{82}$.

L'anonimo autore del libello De rebus bellicis, verso la metà del IV secolo d.C., si preoccupava del fatto che la maestà imperiale venisse lesa quando si rifiutava la sua immagine impressa su monete false ${ }^{83}$. Per tale ragione, perché i falsari commettevano un reato di lesa maestà, ad essi era destinata in genere la pena capitale secondo diverse modalità. Maestro Adamo a Firenze fu arso sul rogo nel 1281; altrove i falsari erano impiccati, decapitati o perfino bolliti; l'anonimo del Derebus bellicis proponeva all'imperatore di deportare in perpetuum tutti i monetieri su un'isola deserta, dove avrebbero dovuto produrre la moneta senza contatto alcuno con il resto del mondo ${ }^{84}$. La proposta dell'anonimo era del tutto impraticabile in quanto le zecche erano site vicinissime alla sede del potere (a Firenze come si è detto nell'area della Loggia dei Lanzi quindi accanto al Palazzo della Signoria), e a Siena dal Duecento la zecca di trovava all'interno dello stesso Palazzo del Comune ${ }^{85}$.

Maestro Adamo è descritto a guisa di liuto, come Avaritia in un Inferno raffigurato nell'affresco del Giudizio Finale nel Duomo di San Gimignano. Avaritia è quindi ingorda di ricchezze. I falsari ingordi per associazione sono metaforicamente puniti nell'arte da demoni che con grandi cucchiai gli colano oro fuso in gola (così sulla facciata scolpita della chiesa di Sainte Foi a Conques, XII secolo). In Russia nel Cinquecento i falsari erano puniti in modo simile con piombo fuso colato nella gola ${ }^{86}$.

Dante stesso, pensando alla pena degli ingordi di oro, si rivolge a Crasso con queste parole: «Crasso, / dilci, che'l sai: di che sapore è l'oro?» (Purg. XX, 116-117). L'episodio si riferisce alla fine del console romano Marco Licinio Crasso il quale fu sconfitto dai Parti a Carre (antica Mesopotamia, oggi Turchia) nel 53 a.C.: secondo la tradizione riferita, Crasso, accecato dal desiderio dell'oro, cadde nella trappola dei Parti che lo uccisero versandogli oro fuso in gola; tuttavia, la frase «Aurum sitisti, aurum bibe» (sei stato ingordo di oro, ora bevilo), attribuita da molti a Cicerone o altri autori antichi, viene citata per la prima volta intorno al 1300 da un anonimo continuatore dello Speculum 
morale di Vincent di Beauvais (III, 7,2), mentre tra gli antichi soltanto Floro (morto nel 145 d.c.) accenna all'oro fuso in gola ma con altre parole ${ }^{87}$.

70 La ricchezza, e le monete come sintesi della ricchezza, sono un pericolo per l'anima e san Francesco di Assisi (1181-1226) proibì ai frati di accettare monete perfino come elemosina: al suo tempo l'uso delle monete metalliche ebbe un grande incremento, riguardando anche la Chiesa e l'amministrazione dei suoi beni ${ }^{88}$.

71 Se nell'ottica di Francesco la moneta, l'avidità e la ricchezza erano un pericolo, non mancano i casi di monete 'buone' anche per l'anima. Monete erano offerte dai pellegrini sugli altari e sulle tombe dei santi, e a volte erano inserite all'interno di queste tombe, a contatto con i corpi. Le ricognizioni le hanno rivelate all'interno della tomba di san Francesco ad Assisi, di san Geminiano a Modena, di sant'Ambrogio a Milano e di molti altri, specialmente nel XII secolo, con una funzione di offerta e forse anche di segno cronologico: la moneta era il manufatto durevole, piccolo e diffuso capace di impersonare il tempo e il luogo dell'offerente ${ }^{89}$. Monete erano offerte nei depositi di fondazione di edifici, come nel caso delle mura della città di Tusculum (Frascati, Roma) rinforzate nel 1183c.: gli scavi sotto un tratto delle mura hanno rinvenuto in una fossa di fondazione un'olla contenente tracce di fichi e uva e sull'esterno gli scheletri di un gallo e una gallina, non macellati ma sacrificati, una bolla di papa Alessandro III e un denaro lucchese; la città fu distrutta nel $1191^{90}$.

Le monete quindi avevano valenze ed usi molteplici, nel male e nel bene, e proprio Dante, dopo aver mostrato dannati falsari, additato come falsario anche Filippo IV di Francia per le sue mutazioni monetarie, osservato l'ingordo Crasso, nel Paradiso usa la metafora della Fede come una moneta nel dialogo con san Pietro (Paradiso XXIV, 82-87). Il santo gli chiede «Dì, buon Cristiano, fatti manifesto: fede che è?»; e Dante risponde argomentando bene così da soddisfarlo:

Così spirò di quello amore acceso;

indi soggiunse: «Assai bene è trascorsa

d'esta moneta già la lega e 'l peso;

ma dimmi se tu l'hai ne la tua borsa».

Ond' io: «Sì ho, sì lucida e sì tonda,

che nel suo conio nulla mi s'inforsa».

San Pietro parla come un cambiavalute che ha verificato le monete ricevute, provandone la qualità del metallo con una pietra di paragone e il peso con una bilancina affidabile; chiede infine a Dante se ne abbia nella sua borsa e lui risponde di averla ben lucida e tonda, senza alcun dubbio sulla bontà del conio ${ }^{91}$. I tre elementi della moneta, precisati da Isidoro di Siviglia, sono qui tutti presenti: metallo, peso e immagine, impressa dal conio: conio è l'impronta che garantisce metallo e peso.

Questa metafora è di grande rilevanza e si ricollega alla metafora agostiniana dell'uomo-nummus Dei, e ci parla della simbologia della moneta e della sacralità della sua produzione, a partire dallo stretto legame tra moneta e sovranità e poi ben dentro le fasi di produzione ${ }^{92}$. Il conio non è uno strumento qualunque, se Dio stesso ne è partecipe ${ }^{93}$. La sacralità delle monete coniate è manifesta nei riti del Venerdì Santo quando i re d'Inghilterra consacravano sull'altare della messa fiorini di Firenze e con il loro metallo realizzavano i 'cramp rings' per guarire dall'epilessia ed in altri usi ${ }^{94}$.

Con questi presupposti si può ora entrare nel vivo del tema del nostro contributo: «femmine da conio». 
76 Ancora recentemente Marco Giola ne ha parlato come di una «enigmatica, e controversa, espressione ${ }^{95}$. Secondo alcuni Dante avrebbe usato 'conio' per indicare 'inganno' citando per tale affermazione la presenza nel volgare fiorentino, e negli statuti comunali ${ }^{96}$. Va detto invece che a Firenze la zecca era praticamente cosa sacra, protetta eleggendo ogni sei mesi nuovi magistrati responsabili (i Signori della zecca); nella processione per la festa di San Giovanni Battista la zecca faceva sfilare un suo carro $^{97}$. Tutti i riferimenti di Dante al termine conio sono senza dubbio legati alla produzione della moneta.

77 Il passo non ha niente di enigmatico conoscendo almeno un po' la fisicità della produzione delle monete e la realtà della trasformazione del metallo in moneta grazie al conio. La forma dei conii medievali e alcune scene di coniazione offrono risposte chiare. Il conio è uno strumento facilmente assimilabile a un membro virile: si tiene con la mano sinistra appoggiato sul tondello 'vergine' (questo è il termine usato) e col martello si colpisce con forza per dare un'impronta senza ritorno: la moneta creata dal conio non può tornare vergine; può solo 'morire' fusa per essere trasformata nuovamente.

78 Il conio violenta, stupra, imprime i tondelli di metallo e il campo dei nemici vinti; il conio-fallo violenta e stupra le 'femmine'.

Figura 1. - Sigillo monetieri Orvieto: la matrice.
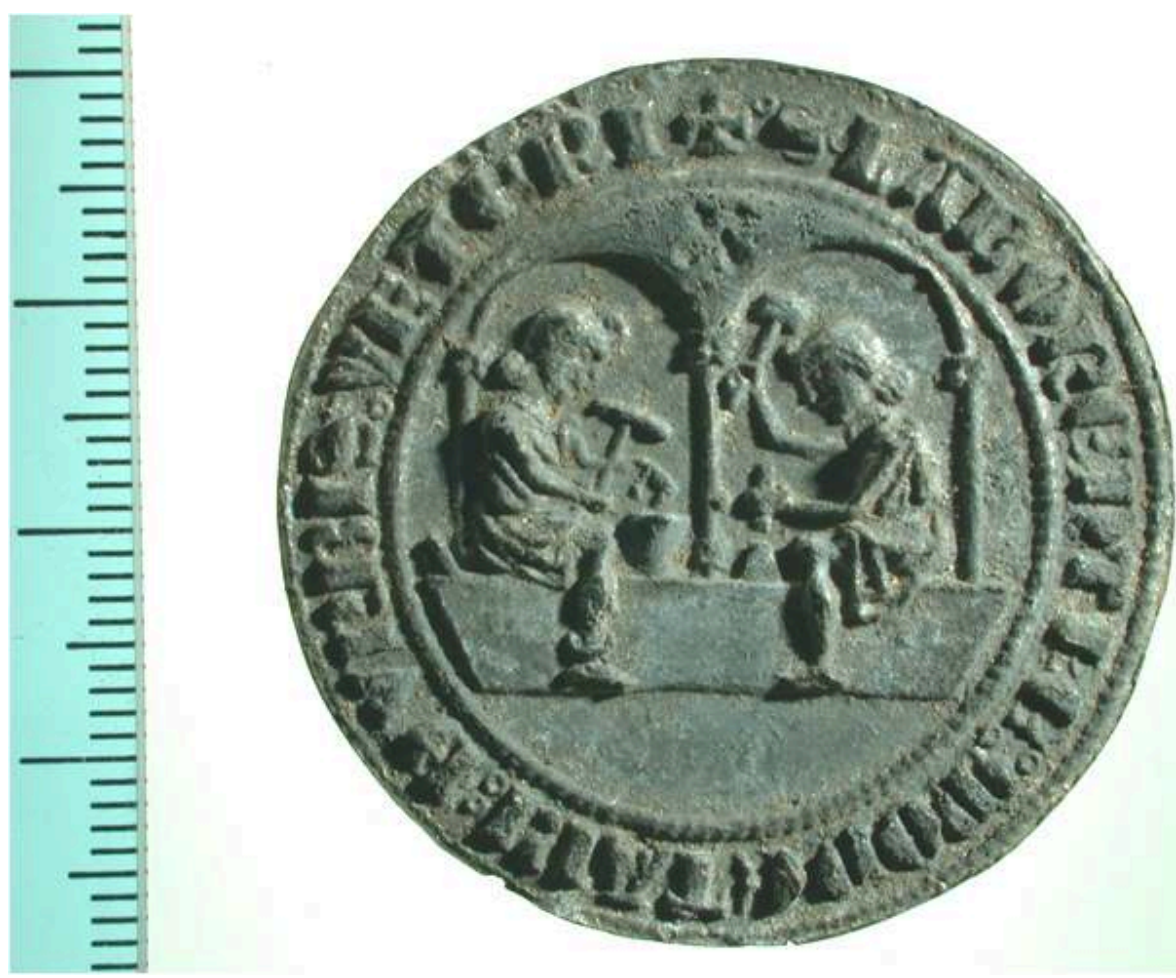

Per gentile concessione del Museo Civico Medievale di Bologna. 
Figura 2. - Sigillo monetieri Orvieto: I'impronta.

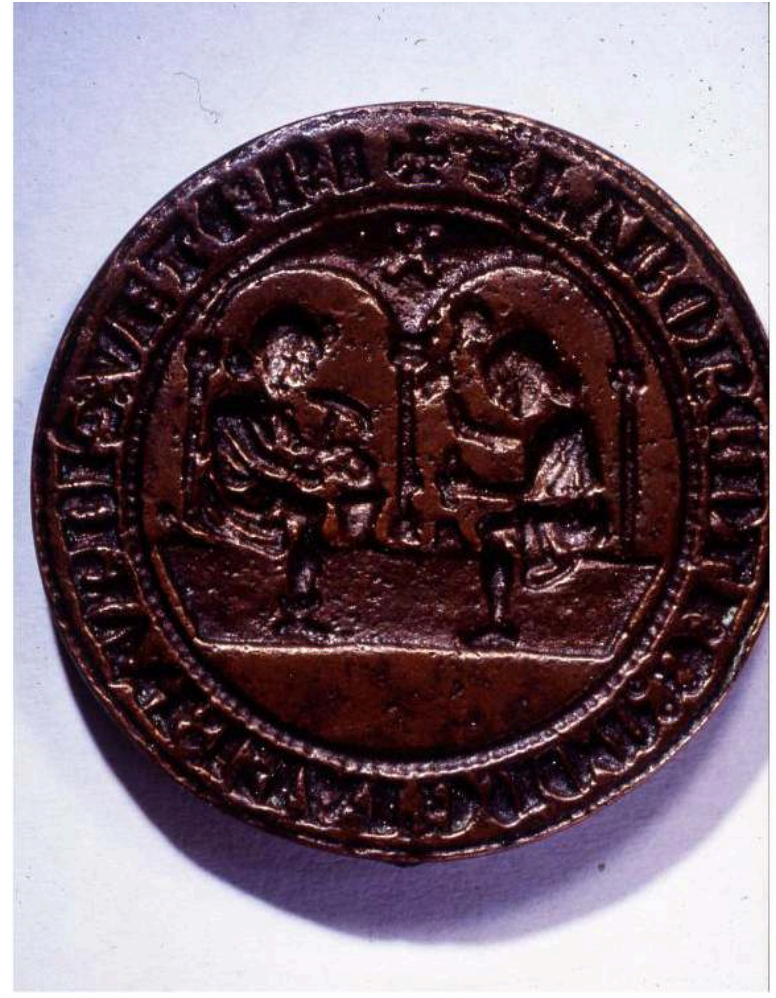

Per gentile concessione del Museo Civico Medievale di Bologna.

\section{NOTE}

1. Così V. Celotto: «la retorica mendace di lenoni e seduttori è dunque sempre perpetrata contro le donne, ma mentre questi ultimi agiscono per un fine personale, che è quello dell'insidia lussuriosa, i ruffiani sono guidati dal guadagno che riscuotono ingannando a beneficio d'altri» (Canto XVIII. La menzogna e il comico, in E. Malato e A. Mazzucchi (a cura di), Lectura Dantis Romana. Cento canti per cento anni. I: Inferno. 2: Canti XVIII-XXXIV, introduzione di E. Malato, prefazione di G. Ravasi, Roma, Salerno, 2013, pp.575-613: p.597), e fra i commentatori antichi già Iacopo Alighieri: «incominciando in questo canto, prima quella [la bolgia, n.d.r.] de' ruffiani si dimostra, nella quale, figurativamente, con contrario andamento d'anime si pone, a dimostrare che due modi contrari la froda e lo 'nganno contra le femmine operata si segnia, cioè per suo propio diletto, o per altrui, per amistà, o per guadagno promesso, e che de' certi diavoli isforzati sieno a significare i lor disii, da' quali continovamente nelle operazioni spronati sono...». Il plurisecolare commento alla Commedia, che comprende anche il testo di Jacopo, è ora leggibile online grazie al Dartmouth Dante Project (<https://dante.dartmouth.edu/>), e cfr. Jacopo Alighieri, Chiose all' «Inferno", a cura di Saverio Bellomo, Padova, Antenore, 1990.

2. Sul carattere inaugurale del canto, cfr. E. Sanguineti, Interpretazione di Malebolge, Firenze, Olschki, 1961; F. Tateo, Il canto XVIII dell'«Inferno», «L'Alighieri. Rassegna bibliografica dantesca», n.s. XII, 1998, pp.33-44 poi, con il titolo La svolta di Malebolge (Inf. XVIII), in Id., Simmetrie 
dantesche, Bari, Palomar, 2001, pp. 69-87; M. Martelli, Canto XVIII, in Lectura Dantis Neapolitana. "Inferno», diretta da P.Giannantonio, Napoli, Loffredo, 1986, pp.301-321; poi, con il titolo Il canto XVIII dell'Inferno, in Id., Ragione e talento. Studi su Dante e Petrarca, a cura di A. Ciadamidaro, premessa di F. Bausi, Cosenza, Falco, 2009, pp. 3-35.

3. Cfr. Z. G. Baranski, Scatology and Obscenity in Dante, in T. Barolini e H. W. Storey (a cura di), Dante for the New Millennium, New York, Fordham University Press, 2003, pp. 259-273 e Id., Inferno XVIII, in E. Pasquini e C. Galli (a cura di), Lectura Dantis Bononiensis, vol. III, Bologna, Bononia University Press, 2014, pp. 81-110.

4. Lo sottolinea S. Bellomo, Nota conclusiva. Canto XVIII, in Dante Alighieri, Inferno, a cura di S. Bellomo, Torino, Einaudi, 2013, p. 296. Cfr. R. Mercuri, Trame metalinguistiche nel canto XVIII dell'Inferno, «Annali dell'Istituto Universitario Orientale di Napoli. Sezione romanza», vol. XXXII, $\mathrm{n}^{\circ} 1,1990$, pp. 201-211.

5. Di «organismo vivente» parla T. Gallarati Scotti, Il canto XVIII dell' «Inferno», in Id., Dante (1947), Milano, Garzanti, 2008, pp. 239-248, e cfr. H. Wayne Storey, Mapping Out the New Poetic Terrain: Malebolge and InfernoXVIII, «Lectura Dantis», $\mathrm{n}^{\circ} \mathrm{IV}$, 1989, pp.30-41; poi, con il titolo "Inferno» XVIII, in T. Wlassics (a cura di), Dante's Divine Comedy. Introductory Readings. I: Inferno, Charlottesville, University of Virginia Press, 1990, pp. 235-246; e cfr. G. Güntert, Canto XVIII, in G. Güntert e M. Picone (a cura di), Lectura Dantis Turicensis. Inferno, Firenze, Cesati, 2000, pp. 243-257.

6. Così Tiziano Zanato interpreta, in maniera a nostro avviso convincente, l'atto di accucciarsi dei dannati della seconda bolgia, non un generico gesto di disperazione, o addirittura un inchino da lusingatori, ma l'urgenza di defecare di chi è condannato a essere immerso nei propri liquami: T. Zanato, Lettura del canto XVIII dell'Inferno, «Per Leggere», nº 6, 2004, pp. 5-47, a p. 36 (e cfr. L. Cappelletti, «Uno sterco / che dagli uman' privadi parea mosso». Inf.XVIII, 113-14: adulatori dissenterici?, «Letteratura Italiana Antica. Rivista annuale di testi e studi», vol. XVIII, 2017, pp. 303-305).

7. Zanato, Lettura, cit., p. 8, che sottolinea come il testo contenga molti hapax, vocaboli rari e rimas caras.

8. Celotto, Canto XVIII, cit. (i passi riportati sono rispettivamente alle pp. 576 e 578).

9. L. Caretti, Etica e retorica nel canto XVIII, in L. Caretti e G. Luti (a cura di), La letteratura italiana per saggi storicamente disposti, vol. I: Le Origini, il Duecento e il Trecento, Milano, Mursia, 1972, pp. 274-279; poi, con il titolo Etica e retorica (Inferno XVIII), in Id., Antichi e moderni. Studi di letteratura italiana, Torino, Einaudi, 1976, pp. 51-70.

10. Di Venedico come centro del canto parla Zanato, Lettura, cit., p.11, che evidenzia poi una «velocizzazione nel trattamento dei personaggi», rilevando che il canto parte con una pausa, alterna poi sommari e scene, e procede poi in maniera sempre più rapida, dunque lo spazio riservato al Caccianemico - e all'episodio che lo riguarda, su cui appunteremo la nostra attenzione - è il maggiore.

11. Preferiscono la dizione «Venetico» F. Sanguineti (Dantis Alagherii, Comedia, a cura di F. Sanguineti, Firenze, Edizioni del Galluzzo, 2001) e Zanato, Lettura, cit., passim.

12. Già incontrato fra i tiranni immersi nel Flegetonte del canto XII, come non manca di sottolineare Bellomo, Introduzione. Canto XVIII, in D. Alighieri, Inferno, cit., p. 283.

13. F. Torraca, Commento alla «Divina Commedia» (1905), a cura di Valerio Marucci, Roma, Salerno, 2008.

14. Così U. Carpi, La nobiltà di Dante, Firenze, Polistampa, 2004, pp. 495-503.

15. Bellomo, Introduzione, cit., p. 284, che prosegue: «Del resto tale accusa segue la menzione degli Estensi, che sono coinvolti da Ugo Capeto nell'esecrazione di Carlo II d'Angiò che mercanteggiò il matrimonio della figlia Beatrice con Azzo (Pg. XX, 79-81). Né lusinghiero pare il riferimento all'altra Beatrice, sorella di Opizzo che, vedova di Nino Visconti, sposò Galeazzo Visconti». Su Venedico, cfr. G. Zaccagnini, Personaggi danteschi in Bologna, «Giornale Storico della Letteratura 
italiana», vol. LXIV, 1914, pp.1-47 (in particolare pp. 27-42). Cfr. P. Chiesa, Canti XVIII-XIX-XX. Il comico e il politico, in S. Invernizzi (a cura di), Esperimenti danteschi. Inferno 2008, prefazione di P. Bocchia, B. Quadrio, C. Sacconaghi e L. Tizzano, Genova / Milano, Marietti, 2009, pp. 141-155.

16. Cfr. S. Carrai, Attraversando le prime bolge. Inferno XVIII, «L'Alighieri. Rassegna dantesca», n.s. XXXVII, 2011, pp. 97-110, a p. 105 (di cui discuteremo più avanti).

17. Inf. XVIII, 46-66. Da ora in poi citiamo il testo da D. Alighieri, La Commedia secondo l'antica vulgata, a cura di G. Petrocchi, vol. 2: Inferno, Firenze, Le Lettere, $1994^{2}$.

18. A. M. Chiavacci Leonardi, nella nota relativa a Inf. XVIII, 66, in D. Alighieri, Commedia, vol. I: Inferno, a cura di A. M. Chiavacci Leonardi, Milano, Mondadori («I Meridiani»), 1991.

19. F. Brambilla Ageno, «Conio» 'Inganno' (Inf. XVIII, 66), in Ead., Studi danteschi, con una premessa di C. Delcorno, Padova, Antenore, 1990, pp.9-12. La Ageno fonda la propria ipotesi su I. Del Lungo, Della interpretazione d'un verso di Dante (Inferno XVIII, 66) rispetto alla storia e della lingua e de' costumi, "Archivio Storico Italiano», s. III, t. XXII, 1875, pp.525-539. Il passo dell'Ottimo commento a suffragio della tesi Del Lungo-Ageno è citato dalla Ageno stessa a p. 9 , nota 2.

20. G. Rigutini, Del vero senso della metafora dantesca «femmine da conio». Studio letterario di G. $R$. Aggiuntovi una Nota filologica di G. Tortoli, Firenze, Tipografia editrice dell'Associazione, 1876.

21. L. Spitzer, Lucca: «sconiato»- florent. «conia», «Archivium romanicum», vol. XI, 1927, pp. 248-250, e L. Passerini, Femmine da conio, «Il Propugnatore», vol. XII, part. II, 1879, pp. 203-213. Entrambi i saggi sono richiamati dalla Ageno, ma l'interpretazione viene scartata («Conio», cit., p. 10).

22. W. Conner, Inf. XVIII, 66 («femmine da conio») and 51 («pungenti salse»), «Italica», vol. XXX, 1955, pp. 95-103.

23. Ageno, «Conio», cit., p. 10.

24. Così Schiaffini, citato dalla Ageno, ivi, p. 10. La recensione di Alfredo Schiaffini si legge negli «Studi Danteschi», vol. XV, 1931, pp. 135-137.

25. N. Pagliaresi, Leggenda di Santo Giosafà, in G. Varanini (a cura di), Cantari religiosi senesi del Trecento, Bari, Laterza, 1965, pp. 5-189, alle pp. 130-131 (e cfr. Ageno, «Conio», cit., p. 11).

26. Sulla bolgia dei falsari, e su questo episodio in particolare, cfr. A. Bruni, Per il canto XXX dell'Inferno, «L'Alighieri. Rassegna dantesca», n.s. XXXVIII, 2011, pp. 91-108, ora riveduto, con il titolo Canto XXX. Duello di parole, duello di mano nel diverbio fra Maestro Adamo e Sinone, in Lectura Dantis Romana, cit., pp. 939-960.

27. Guglielmo Maramauro, Expositione sopra l'Inferno di Dante Alligieri, a cura di P.G. Pisoni e S. Bellomo, Padova, Antenore, 1998 (il testo si legge online in Dartmouth Dante Project, cit.).

28. Benevenuti de Rambaldis de Imola Comentum super Dantis Aldigherij Comoediam, nunc primum integre in lucem editum sumptibus Guilielmi Warren Vernon, curante Jacobo Philippo Lacaita, Florentiae, G. Barbèra, 1887, 5 voll. (il testo si legge online in Dartmouth Dante Project, cit.).

29. Ottimo commento alla 'Commedia', a cura di G. B. Boccardo, M. Corrado e V. Celotto, Roma, Salerno Editrice, 2018, 3 voll. (il testo si legge online in Dartmouth Dante Project, cit., e cfr. L'ultima forma dell'Ottimo commento. Chiose sopra la Comedia di Dante Alleghieri fiorentino tracte da diversi ghiosatori. Inferno, a cura di C. Di Fonzo, Ravenna, Longo, 2008).

30. Commento di Francesco da Buti sopra la «Divina Commedia» di Dante Alighieri, a cura di C. Giannini, Pisa, Nistri, 1858-1862, 3 voll. (il testo si legge online in Dartmouth Dante Project, cit.).

31. Commento alla Divina Commedia d'Anonimo Fiorentino del secolo XIV, ora per la prima volta stampato a cura di P. Fanfani, Bologna, Romagnoli, 1866-1874, 3 voll. (il testo si legge online in Dartmouth Dante Project, cit.).

32. Lo Inferno della Commedia di Dante Alighieri col comento di Guiniforto delli Bargigi, con introduzione e note dell'avv. G. Zacheroni, Marsilia / Firenze, Leopoldo Mossy e Giuseppe Molini, 1838 (il testo si legge online in Dartmouth Dante Project, cit.).

33. Sanguineti, Interpretazione, cit., p. 16. 
34. Baranski, Inferno XVIII, cit., p. 81.

35. Zanato, Lectura, cit., p. 8.

36. Baranski, Inferno XVIII, cit., p. 82.

37. Ibid.

38. Ibid.

39. Ivi, p. 84 e Id., Scatology and Obscenity, cit., passim.

40. Baranski, Inferno XVIII, cit., p. 84.

41. Ivi, pp. 91-92.

42. Il riferimento è ovviamente a M. Bachtin, L'opera di Rabelais e la cultura popolare. Riso, carnevale e festa nella tradizione medievale e rinascimentale (1965), trad. it. Torino, Einaudi, 2001.

43. Baranski, Inferno XVIII, cit., p. 97.

44. Ivi, p. 98.

45. Ibid., e C. Casagrande e S. Vecchio, I peccati della lingua: Disciplina ed etica della parola nella cultura medievale, Roma, Istituto della Enciclopedia italiana, 1987.

46. Baranski, Inferno XVIII, cit., p. 99.

47. Bellomo, in D. Alighieri, Inferno, a cura di S. Bellomo, cit., p. 290, n. al v. 66.

48. Il Ristorato, poema inedito in terza rima del secolo XIV di Ristoro Canigiani, a cura di L. Razzolini, Firenze, Tipografia Galileiana, 1847.

49. Ivi, vv. 7-12, p. 96.

50. Oggi interamente online: <http://tlio.ovi.cnr.it>.

51. B. Angioini, La Canzona de' tagliatori di legname, in C. Singleton (a cura di), Canti Carnascialeschi del Rinascimento, Bari, Laterza, 1936, p. 217.

52. M. Costa, Li buffoni. Prologo, vv. 8-14, in S. Ferrone (a cura di), Commedie dell'Arte, Milano, Mursia, 1986, vol. II, p. 245.

53. Celotto, Canto XVIII, cit., p. 600.

54. «Dante non ha presente, in questa circostanza, il testo terenziano (ma ciò non è sufficiente per escluderne la conoscenza, data la sua diffusione: Villa), ma un passo di Cicerone, De amicitia, XXVI, 98 [...]. Dove, non solo il testo consente di attribuire direttamente a Taide la risposta [...] ma anche addita queste parole come particolarmente adulatorie» (Bellomo, Introduzione, cit., p. 285). Il personaggio di Taide diventa esemplare di prostituta adulatrice grazie al Liber Esopi, versione in distici elegiaci, composta da Gualtiero Anglico, di una raccolta latina in prosa di epoca carolingia (G. Padoan, Il «Liber Esopi» e due episodi dell'Inferno, «Studi danteschi», vol. XLI, 1964, pp. 75-102, ora in Id., Il pio Enea, l'empio Ulisse. Tradizione classica e intendimento medievale in Dante, Ravenna, Longo, 1977, pp.151-169, e cfr. C. Villa, La «Lectura Terentii». Da Ildemaro a Francesco Petrarca, Padova, Antenore, 1984).

55. Zanato, Lettura, cit., p. 40.

56. Carrai, Attraversando, cit., p. 105.

57. Per una panoramica critica aggiornata sulla figura di Piccarda, cfr. L. Battaglia Ricci, Piccarda, o della carità: lettura del terzo canto del Paradiso, «Filologia e critica», vol. XIV, 1989, pp. 27-70, ora in Lectura Dantis Romana, cit., vol. III: Paradiso, 1. Canti I-XVII, Roma, Salerno, 2015, pp. 85-110.

58. Carrai, Attraversando, cit., pp. 97-98: la definizione di Dante come «crudele» è di G. Bertoni, I lenoni e gli adulatori (Inferno, c. XVIII), in Id., Cinque letture dantesche, Modena, Società Tipografica Modenese, 1933, pp. 32-33. Carrai insiste sul fatto che «il deplorevole esempio di Venèdico Caccianemico, che vendette la propria sorella per interesse personale, e quello degli adulatori Alessio Interminelli e Taide evocano nella fantasia di Dante pene ferine e umilianti, vale a dire le continue sferzate distribuite dai diavoli e il giacere nei propri liquami» (ivi, p. 99).

59. Ibid.

60. Ivi, p. 100. Carrai parla efficacemente di «estetica del brutto dello sporco e del cattivo», ricordando il film di Ettore Scola (p. 104). 
61. «Ciò che accomuna le quattro figure di peccatori riunite in questo canto è infatti l'avere gabbato e sfruttato l'amore: sia a fini altrui che propri, nel mondo moderno come in quello antico» (ivi, p. 102).

62. Bellomo, Introduzione, cit., p. 284.

63. Carrai, Attraversando, cit., p. 101.

64. La fonte è G. Villani, Cronica, IX, 46. L'Interminelli è presente a Firenze, forse proprio come esponente dei Bianchi, fino al novembre 1299 (Carpi, La nobiltà, cit., p. 504), e forse in quest'occasione Dante lo incontra e conosce: Bellomo, Introduzione, cit., p. 285.

65. Ibid.

66. Zanato, Lettura, cit., p. 20.

67. Ibid.

68. Ivi, p. 26. Il richiamo è ad A. Pagliaro, Commento incompiuto all'Inferno di Dante. Canti I-XXVI, a cura di G. Lombardo, Roma, Herder Editrice e Libreria, 1999.

69. E. Raimondi, Una città nell'inferno dantesco (1967), in Id., Metafora e storia. Studi su Dante e Petrarca, Torino, Einaudi, 1970, pp. 39-63.

70. Il primo significato deriva dal greco kóvıoৎ «polveroso», e riguarda termini scientifici composti estranei al nostro tema.

71. Esempi di questo tipo sono i fiorini d'oro di Firenze nei tesori scoperti a Montella (Avellino) e a Siracusa; si vedano L. Travaini e M. Broggini (a cura di), Il tesoro di Montella (Avellino). Fiorini e ducati d'oro occultati nella metà del Trecento, Roma, Quasar, 2016; S. Locatelli, Florins and Ducats in the Kingdom of Sicily-Aragon. The Syracuse Hoard (1313-c. 1369), «Numismatic Chronicle», vol. 179, 2019, pp. 299-340.

72. Per un inventario dei conii antichi e medievali, cfr. L. Travaini, I conii e le zecche, in L. Travaini e A. Bolis (a cura di), Conii e scene di coniazione, Roma, Quasar, 2007, pp. 27-66. Per le collezioni di conii e punzoni di età moderna: R. Doty, Tecnologia delle zecche italiane: nota sulle collezioni di conii nei musei italiani, in L. Travaini (a cura di), Le zecche italiane fino all'Unità, Roma, Istituto Poligrafico dello Stato, 2011, pp. 293-308.

73. Su quest'ultimo aspetto in particolare, si veda L. Travaini, Monete e sangue, in Id. (a cura di), Valori e disvalori simbolici delle monete nel medioevo. I Trenta denari di Giuda, Roma, Quasar, 2009, pp. 231-248, a pp. 240-241.

74. Dettagli sui provvedimenti in materia da parte di alcune zecche italiane in L. Travaini, Le zecche italiane, in Id. (a cura di), Le zecche italiane fino all'Unità, Roma, Istituto Poligrafico dello Stato, 2011, pp. 31-122, a pp. 78-81.

75. Zecca deriva dall'arabo sikka: per la storia del termine, si vedano L. Travaini, Mints as Volcanoes: Fire and Technology, in M. Caccamo Caltabiano (a cura di), XV International Numismatic Congress, Taormina 2015, Proceedings, 2 voll., Roma / Messina, Arbor Sapientiae, 2017, vol. I, pp. 30-35. Si veda per i caratteri generali L. Travaini, Le zecche italiane, cit., pp. 31-122.

76. Per tutto, si veda P.Grierson, Coniazioni per dispetto nell'Italia medievale, «Numismatica e Antichità Classiche. Quaderni Ticinesi», nº 8, 1979, pp. 345-358 (riprodotto in P. Grierson, Scritti storici e numismatici, a cura di E. A. Arslan e L. Travaini, Spoleto, CISAM (Collectanea 15), 2001, pp. 303-358). Qui l'autore approfondì il tema trattato da G. Ruggero, Monete battute in campo dai Fiorentini e dai Pisani, «Rivista Italia di Numismatica», $n^{\circ} 20,1907$, pp. 402-403.

77. «In numismate tria quaerentur: metallum, figura et pondus. Si ex his aliquid defuerit nomisma non eriit»: Isidori Hispalensis Episcopi Etymologiarum Sive Originum Libri XX, a cura di W. Lindsay, Oxford (UK), Clarendon Press, 1911, t. 1, 1. XVI, 18.12.

78. Non si approfondisce qui il complesso tema della metrologia monetaria; si rinvia a W. R. Day Jr, Metrologia monetaria, in L. Travaini (a cura di), Le zecche italiane fino all'Unità, Roma, Istituto Poligrafico dello Stato, 2011, pp. 321-331, e alle tabelle di conversione carato e once in millesimi di oro e argento in L. Travaini, Monete mercanti e matematica. Le monete medievali nei trattati di 
aritmetica e nei libri di mercatura, Roma, Jouvence, 2003, pp. 314-316 (seconda edizione ampliata con nuove liste inedite, Milano, Jouvence, 2020, pp. 314-316).

79. La divisione del lavoro era più articolata di quanto sopra elencato: si veda per maggiori particolari L. Travaini, Le zecche italiane, cit., pp. 66-78.

80. Emilio Bigi, voce: Maestro Adamo nell' Enciclopedia dantesca, 1970, on-line, <https:// www.treccani.it/enciclopedia/adamo_res-

a2d44ac8-87ee-11dc-8e9d-0016357eee51_(Enciclopedia-Dantesca)/>:

«I commentatori antichi non forniscono notizie più precise sul dannato, a parte alcune contrastanti indicazioni sulla sua patria («de Casentino» per il Bambagliuoli; «bolognese» nelle chiose Selmi; «decivitate opulenta Brixiae» [Brescia] secondo Benvenuto). Un contributo importante all'identificazione del falsario si trae invece da un atto rogato a Bologna il 28 ottobre 1277 (pubblicato dal Tarlazzi nel 1869, ma posto in evidenza dal Palmieri nel 1889), nel quale si parla di un «magistro Adam de Anglia familiare comitum de Romena». Non ci sono, in conclusione, ragioni serie per mettere in dubbio l'origine, o almeno la provenienza, dall'Inghilterra del maestro A.; ed è certo, in ogni caso, che egli non fu un semplice artigiano, ma appunto un magister, «termine tecnico del mondo universitario, grado sinonimo di dottore», addottorato in una facoltà che «più che la medica, ben probabilmente è ancora quella delle Artes, fra le quali vennero classificate le cosiddette scienze naturali» (cfr. G.Contini, Sul XXX dell'Inferno, «Paragone», vol. XLIV, 1953, pp. 3-13).

81. L. Travaini, Monete mercanti e matematica, cit., p. 114.

82. Un fiorino di Firenze falso con nucleo di rame e perduta doratura superficiale fa parte del tesoro di Montella.

83. A. Giardina (a cura di), Le cose della guerra, Verona, Fondazione Lorenzo Valla-Mondadori, 1989, pp. 14-15.

84. Sulle pene per falsa moneta nel medioevo europeo, cfr. C. De Graaf, Den valscherden ketel? Bestraffing van muntmisdrijven in Holland, Zeeland en Utrecht ca 1300-ca 1600, «Jaarboek voor Munten Penningkunde», $n^{\circ} 82,1995$, pp. 77-179. Per il delitto di lesa maestà nella tarda antichità e oltre, si veda anche F. Carlà, Dal perpetuum exilium al taglio della mano: falso in moneta e maiestas nel diritto tardoromano, bizantino e romano-germanico, in J. Chameroy e P.-M. Guihard (a cura di), Produktion und Recyceln von Münzen in der Spätantike: Produire et recycler la monnaie au Bas-Empire; 1. Internationales Numismatikertreffen; $1^{\text {res }}$ Rencontres internationales de numismatique (Mainz, 15-16 maggio 2014), Mainz, Römisch-Germanisches Zentralmuseum, 2016, pp. 29-46; anche L. Travaini, I Trenta denari di Giuda. Storia di reliquie impreviste nell'Europa medievale e moderna, Roma, Viella, 2020, p. 29.

85. L. Travaini, Sedi di zecca nell'Italia medievale, in Iluoghi della moneta. Le sedi delle zecche dall'antichità all'età moderna, Atti del convegno internazionale (Milano, 22-23 ottobre 1999), Milano, Comune di Milano, 2001, pp. 69-85.

86. La pena in Russia è citata da C. De Graaf, Den valscherden ketel?, cit. Per l'affresco ed altre raffigurazioni simili, si veda L. Travaini, Le zecche illustrate: iconografia e interpretazione, in L. Travaini e A. Bolis (a cura di), Conii e scene di coniazione, Roma, Quasar, 2007, pp. 259-299.

87. Si veda P. Garbini, L'«exemplum» di Crasso: Purgatorio, XX, 116-17, «Filologia e critica», vol. 16, 1991, pp. 272-276.

Il tema è trattato con altri esempi in L. Travaini, I Trenta denari, cit., p. 23.

88. Si vedano i contributi in M. Benedetti e T. Subini (a cura di), Francesco d'Assisi. Storia, arte, mito, Roma, Carocci, 2019, e in particolare per le monete: L. Travaini, San Francesco nella ricerca numismatica: iconografia, e non solo, pp. 73-85.

89. Sulle monete come segno di identità, si vedano L. Travaini, Coins and Identity: From the Mint to Paradise, in R. Naismith (a cura di), Money and Coinage in the Middle Ages, Leiden, Boston, Brill, 2018 (Reading Medieval Source, 1), pp. 320-349; Ead, Saints, Sinners and ... a Cow: Offerings, Alms and Tokens of Memory, in G. Gasper e S. Gullbekk (a cura di), Money and the Church in Medieval Europe, 
1000-1200: Practice, Morality and Thought, Farnham, Ashgate, 2015 (Religion and Money in the Middle Ages), pp. 209-221. Monete furono usate nel 1406 come segno lasciapassare alle porte di Reggio Emilia, Parma e delle rocche nei rispettivi territori: L. Travaini e M. Bazzini, Signa Uguccionis: monete come prova di identità tra Parma e Reggio Emilia nel 1409, «Revue Numismatique», vol. 172, 2015, pp. 391-449.

90. G. Mandatori, Un deposito di fondazione medievale dalle mura di Tusculum (XII secolo), «Numismatica e Antichità Classiche. Quaderni Ticinesi», vol. 46, 2017, pp. 175-195.

91. Par. XXIV, 82-87: E. Edwards, Money and Literature, in Money and Coinage in the Middle Ages, cit., pp. 264-286, a p. 272; G. Alonzo, Numismatica dantesca. La Commedia tra meledizione e santificazione della moneta, in F. Spera (a cura di), Stella Forte. Studi danteschi, Napoli, D'Auria editore, 2010, pp. 81-105, a pp. 98-100.

92. Molti aspetti sono trattati in L. Travaini, Sacra Moneta. Mints and Divinity: Purity, Miracles and Powers, in N. M. Burström e G. T. Ingvardson (a cura di), Divina Moneta. Coins in Religion and Ritual, Abingdon, Routledge, 2018 (Religion and Money in the Middle Ages, 2), pp. 174-189. Qui anche la recente discussione sull'etimologia di "moneta" da "moneo" (p. 185, nota 5).

93. Per la metafora agostiniana: P. Radici Colace, Il Dio monetiere, in Conii e scene di coniazione, cit., pp. 13-25; G. Todeschini, Il denaro come fattore di inclusione o di esclusione: da Graziano a Cusano, in R. Lambertini e L. Sileo (a cura di), I Beni di questo mondo. Teorie etico-economiche nel laboratorio dell'Europa medievale, Porto, Fédération internationale des Instituts d'études médiévales / Gabinete de Filosofia Medieval, Faculdade de Letras U.P., 2010, pp.17-36. L. Travaini, I Trenta denari, cit., p. 28. Per la metafora del sigillo: B. Bedos-Rezak, Medieval Identity: A Sign and a Concept, «American Historical Review», vol. 105, n5 5, 2000, pp. 1489-1533 (riprodotto in Medieval Coins and Seals, pp. 65-80). Per il rapporto tra ostie, pane e monete: L. Travaini, Coins as Bread. Bread as Coins, «Numismatic Chronicle», vol. 173, 2013, pp. 187-200.

94. Per questo e altri rituali che implicano l'uso di monete sintesi e bibliografia in Travaini, I Trenta denari, cit., capitolo 1.

95. M. Giola, Dante e la lessicografia mediolatina. Le Derivationes di Uguccione da Pisa tra la Commedia e i suoi antichi commentatori: un esperimento di spoglio, «Versants», vol. 58, nº 2, 2011, p. 201.

96. Ageno, Conio, cit.

97. Travaini, Sacra Moneta, cit. p.176; per il carro del 1362: M. Bernocchi, Le monete della Repubblica Fiorentina, vol. I: Il libro della zecca di Firenze, Firenze, Olschki, 1974, p. LXxI. Sulla zecca di Firenze, si veda W. R. Day Jr, Antiquity, Rome and Florence: Coinage and Transmissions across Time and Space, in C. Bolgia, R. McKitterick e J. Osborne (a cura di), Rome across Time and Space: Cultural Transmission and the Exchange of Ideas, c. 500-1400, Cambridge, 2011, pp. 237-262.

\section{RIASSUNTI}

L'espressione «femmine da conio» nel canto XVIII dell'Inferno ha avuto interpretazioni molto diverse, su cui i commentatori antichi e quelli moderni non si sono mai accordati. In questo saggio si propone una soluzione, analizzando l'uso che Dante fa della parola «conio» e il contesto del canto XVIII, nonché il tema della prostituzione e dello sfruttamento delle donne da parte degli uomini condannati in Malebolge. Vengono infine spiegate le tecniche di coniazione della moneta nel tardo Medioevo, e le immagini che queste tecniche potevano suscitare nei primi 
lettori della Commedia. La metafora in questo modo diventa chiara: si comprende il senso dell'espressione usata da Dante e il significato complessivo del canto XVIII.

L'expression «femmine da conio » dans le chant XVIII de l'Enfer de Dante a eu des interprétations très différentes, sur lesquelles les commentateurs anciens et modernes n'ont jamais été d'accord. Dans cet essai, on propose une solution, en analysant l'usage que Dante fait du mot «conio » et le contexte du chant XVIII : le thème de la prostitution et de l'exploitation des femmes par les hommes condamnés à l'enfer. Enfin, on explique les techniques de frappe des monnaies à la fin du Moyen Âge et les images que ces techniques pouvaient susciter chez les premiers lecteurs de la Divine Comédie. La métaphore devient ainsi claire : on comprend le sens de l'expression utilisée par Dante et la signification globale du chant XVIII.

The expression "femmine da conio" in Canto XVIII of Dante's Inferno has had very different interpretations, by ancient and modern commentators. In this essay we propose a solution, analyzing the use of the word "conio" in Dante's Commedia and the context of Canto XVIII; the theme of prostitution and of women's exploitation by men condemned to hell. Finally, we examine mint technology in the late Middle Ages, and the images that these techniques could suggest to the first readers of the Commedia. The metaphor thus becomes clear: we can understand the meaning of the expression used by Dante, and the overall meaning of Canto XVIII.

\section{INDICE}

Mots-clés : prostitution, frappe, monnaie et technologie, « conio » (coin)

Parole chiave : prostituzione, zecca e tecnologia, coniazione, moneta, "conio"

Keywords : prostitution, coinage, mint technologie, "conio" (die)

\section{AUTORI}

\section{SEBASTIANA NOBILI}

Claudia Sebastiana Nobili è professoressa ordinaria di Letteratura italiana all'Università di Bologna dove si occupa di letteratura due-trecentesca - in particolare di Dante e Boccaccio e di narrativa e teatro nel primo Novecento. Ha pubblicato, tra l'altro, l'edizione commentata della Cronica di Salimbene da Parma (2002), e le monografie «La materia del sogno». Pirandello tra racconto e visione (2007), Giovanni Boccaccio (2014) e La consolazione della letteratura. Un itinerario fra Dante e Boccaccio (2017).

Claudia Sebastiana Nobili est professeure de littérature italienne à l'Université de Bologne, où elle travaille sur la littérature des XIII ${ }^{\mathrm{e}}$ et XIv ${ }^{\mathrm{e}}$ siècles - en particulier Dante et Boccace - et sur la fiction et le théâtre au début du $\mathrm{xx}^{\mathrm{e}}$ siècle. Elle a publié, entre autres, l'édition annotée de la Cronica de Salimbene da Parma (2002), et les monographies La materia del sogno. Pirandello tra racconto e visione (2007), Giovanni Boccaccio (2014) et La consolazione della letteratura. Un itinéraire entre Dante et Boccace (2017).

sebastiana.nobili@unibo.it

\section{LUCIA TRAVAINI}

Lucia Travaini è professoressa associata di Numismatica all'Università degli Studi di Milano e studia le monete medievali e moderne sotto diversi profili: circolazione, produzione, iconografia e usi rituali. Tra i suoi libri: I Trenta denari di Giuda. Storia di reliquie impreviste nell'Europa medievale 
e moderna (2020); Monete mercanti e matematica. Le monete medievali nei trattati di aritmetica e nei libri di mercatura (2020); Le zecche italiane fino all'Unità (ed. 2011); I capelli di Carlo il Calvo. Indagine sul ritratto monetale nell'Europa medievale (2013).

Lucia Travaini est professeur associé de numismatique à l'Université d'État de Milan et étudie les monnaies médiévales et modernes sous différents angles : circulation, production, iconographie et usages rituels. Parmi ses livres : I Trenta denari di Giuda. Storia di reliquie impreviste nell'Europa medievale e moderna (2020); Monete mercanti e matematica. Le monete medievali nei trattati di aritmetica e nei libri di mercatura (2020); Le zecche italiane fino all'Unità (ed. 2011) ; I capelli di Carlo il Calvo. Indagine sul ritratto monetale nell'Europa medievale (2013).

travaini.lucia@gmail.com 